\title{
Les partis anti-système dans la Roumanie post-communiste
} Antoine Roger

\section{Citer ce document / Cite this document :}

Roger Antoine. Les partis anti-système dans la Roumanie post-communiste. In: Revue d'études comparatives Est-Ouest, vol. 31, 2000, n². pp. 101-136;

doi : https://doi.org/10.3406/receo.2000.3027

https://www.persee.fr/doc/receo_0338-0599_2000_num_31_2_3027

Fichier pdf généré le 12/04/2018 


\begin{abstract}
Three Romanian political parties (PSM, PUNR and PRM) have categorically opposed the economic reforms introduced since 1990. Not satisfied with filling the "tribuni- tian function" defined by Georges Lavau, they intend to overthrow the existing political order. The concept of an antisystem party sheds light on the dual internal structure and the "logic of doctrinal doubling" inside each of these parties. A legalist faction wants to infiltrate the political system so as to undermine it from the inside. It is seeking an alliance with the PDSR, a party that, pursuing a majority in Parliament, opposes accelerating the liberalization of the economy but does not refuse the very principle of reforms. Meanwhile, a seditious faction intends to launch a frontal attack on the political system without becoming involved with any other formation. The equilibrium between these two factions varies from one party to another and over time. Relations between the PDSR and the antisystem parties are strained. Whether in office (19921996) or in the opposition (1996-...), the PDSR has to rely on other political forces. It seeks the support of legalist factions, but cannot, without compromising itself, accept an alliance with the seditious factions. This tension has altered its political program; the anti- system parties thus exert a parasitical power.
\end{abstract}

\title{
Résumé
}

Trois partis politiques roumains - le PSM, le PUNR et le PRM - s'opposent catégoriquement aux réformes engagées depuis 1990 : ils ne se contentent pas d'exercer une " fonction tribunitienne »au sens où l'entend Georges Lavau mais aspirent à renverser l'ordre établi. En nous appuyant sur la notion de parti anti-système, nous pouvons mettre en évidence le caractère bifide de leur structure interne. Dans chacun des cas, une logique de dédoublement doctrinal est à l'œuvre. Une faction légaliste souhaite pénétrer le système politique pour le miner de l'intérieur ; elle recherche une alliance avec le PDSR, parti à vocation majoritaire qui s'oppose à une libéralisation accélérée de l'économie sans remettre en cause le principe même des réformes. Une faction séditieuse entend attaquer le système politique frontalement, sans se commettre avec aucune formation extérieure. Les rapports de force entre les deux factions diffèrent d'un parti à l'autre et sont du reste évolutifs. Le PDSR entretient des relations tendues avec les partis anti-système. Qu'il soit au pouvoir (1992-1996) ou dans l'opposition (1996-...), il doit s'assurer de quelques contreforts politiques. II sollicite le soutien des factions légalistes mais ne peut accepter sans risque de compromission une alliance avec les factions séditieuses abritées par les mêmes formations. Son programme politique est adultéré par cette tension. Les partis anti-système exercent ainsi un pouvoir parasitaire. 


\title{
LES PARTIS ANTI-SYSTÈME DANS LA ROUMANIE POST-COMMUNISTE
}

\author{
Antoine ROGER *
}

\begin{abstract}
RÉsumÉ : Trois partis politiques roumains - le PSM, le PUNR et le PRM - s'opposent catégoriquement aux réformes engagées depuis 1990 : ils ne se contentent pas d'exercer une « fonction tribunitienne » au sens où l'entend Georges Lavau mais aspirent à renverser l'ordre établi. En nous appuyant sur la notion de parti anti-système, nous pouvons mettre en évidence le caractère bifide de leur structure interne. Dans chacun des cas, une logique de dédoublement doctrinal est à l'œuvre. Une faction légaliste souhaite pénétrer le système politique pour le miner de l'intérieur ; elle recherche une alliance avec le PDSR, parti à vocation majoritaire qui s'oppose à une libéralisation accélérée de l'économie sans remettre en cause le principe même des réformes. Une faction séditieuse entend attaquer le système politique frontalement, sans se commettre avec aucune formation extérieure. Les rapports de force entre les deux factions diffèrent d'un parti à l'autre et sont du reste évolutifs. Le PDSR entretient des relations tendues avec les partis anti-système. Qu'il soit au pouvoir (1992-1996) ou dans l'opposition $(1996-. .$.$) , il doit s'assurer de quelques contreforts politiques. Il sollicite le soutien des fac-$ tions légalistes mais ne peut accepter sans risque de compromission une alliance avec les factions séditieuses abritées par les mêmes formations. Son programme politique est adultéré par cette tension. Les partis anti-système exercent ainsi un pouvoir parasitaire.
\end{abstract}

ABSTRACT : Three Romanian political parties (PSM, PUNR and PRM) have categorically opposed the economic reforms introduced since 1990. Not satisfied with filling the "tribunitian function" defined by Georges I avau, they intend to overthrow the existing political order. The concept of an antisystem party sheds light on the dual internal structure and the "logic of doctrinal doubling" inside each of these parties. A legalist faction wants to infiltrate the political system so as to undermine it from the inside. It is seeking an alliance with the PDSR, a party that, pursuing a majority in Parliament, opposes accelerating the liberalization of the economy but does not refuse the very principle of reforms. Meanwhile, a seditious faction intends to launch a frontal attack on the political system without becoming involved with any other formation. The equilibrium between these two factions varies from one party to another and over time. Relations between the PDSR and the antisystem parties are strained. Whether in office (1992-1996) or in the opposition (1996-...), the PDSR has to rely on other political forces. It seeks the support of legalist factions, but cannot, without compromising itself, accept an alliance with the seditious factions. This tension has altered its political program; the antisystem parties thus exert a parasitical power.

Depuis 1990, la libéralisation économique de la Roumanie obéit à un rythme des plus heurté. Pour pallier le défaut de capitaux autochtones, les autorités sont dans l'obligation de solliciter des crédits internationaux. Elles signent régulière-

* ATER en science politique à l'Institut d'Études Politiques de Bordeaux (Allée Ausone, B.P. 101, Domaine universitaire, 33405 Talence Cedex). 
ment des accords avec le Fond Monétaire International. En contrepartie des subsides obtenus, elles promettent de se conformer à un régime économique draconien (réduction du déficit budgétaire, liquidation des entreprises publiques déficitaires...) mais elles peinent à respecter leurs engagements. Les créditeurs internationaux leur adressent vainement quelques avertissements sans frais, puis finissent par les semoncer et par interrompre leurs versements. Après que les dirigeants roumains ont montré quelques signes de contrition, les négociations reprennent : un nouvel accord est conclu, qui ne fait que préluder à une nouvelle crise... Le mouvement cyclique ainsi décrit n'affecte pas seulement la sphère économique mais influe aussi très largement sur la vie politique roumaine.

Formé en décembre 1989 dans le prolongement des manifestations antiCeausescu, le Front de Salut National (Frontul Salvarii Nationale - FSN) devient en 1990 un parti politique. Il obtient des succès électoraux retentissants, recueillant $66,31 \%$ des suffrages aux élections législatives et conquérant 263 sièges sur 387 au sein de la Grande Assemblée Nationale. Ion Iliescu est élu président de la République au premier tour de scrutin, avec $87,07 \%$ des voix. Ces résultats ne doivent pas être interprétés comme une marque d'unanimisme. Le FSN est encore une formation composite. Il abrite plusieurs partis en devenir. Si les lignes de clivages restent assez brouillées dans un premier temps, deux factions en viennent rapidement à s'affronter. L'une - dirigée par le Premier ministre Petre Roman - prône une libéralisation accélérée de l'économie roumaine et une observation totale des recommandations formulées par les créditeurs internationaux ; l'autre - fidèle au président Iliescu - préconise d'emprunter une voie économique originale, adaptée aux « spécificités roumaines ». Ces programmes antagoniques s'appuient sur deux représentations de l'intérêt national. Petre Roman et ses partisans admettent que les réformes économiques sont socialement douloureuses, mais jugent qu'elles sont incontournables et qu'il est inutile de les différer ; ils estiment qu'elles doivent immuniser la société roumaine contre des souffrances plus vives encore. Ion Iliescu et ses disciples soutiennent qu'en s'efforçant de reproduire fidèlement les schémas économiques occidentaux, la Roumanie se condamne à un rôle subalterne ; ils concèdent que des réformes doivent être engagées pour obtenir les crédits du FMI mais entendent qu'elles soient limitées au strict minimum et ne leur reconnaissent qu'une valeur instrumentale.

Les partisans d'une libéralisation accélérée accèdent rapidement aux commandes. Après que le FSN a remporté triomphalement les élections, un mouvement protestataire s'organise à Bucarest : les étudiants occupent la place de l'Université et réclament la démission des «néo-communistes » investis par les urnes. Après plusieurs semaines de troubles, les mineurs de la vallée du Jiu gagnent la capitale et s'en prennent violemment aux manifestants. Le FSN cautionne cette répression et s'attire aussitôt l'opprobre des observateurs internationaux. Soucieux de redorer son image extérieure, il met en avant les personnalités qui rassurent les organismes créditeurs. Petre Roman peut s'entourer de fídèles au gouvernement et mener la politique de son choix. Il se conforme aux recom- 
mandations du FMI et de la Banque mondiale. Ce faisant, il s'aliène la partie la plus fragilisée du salariat. Les adversaires du Premier ministre relaient le mécontentement populaire. En 1991, les mineurs leur apportent un concours actif : ils se déplacent de nouveau à Bucarest et y manifestent violemment contre les réformes économiques engagées. Petre Roman est poussé à la démission. Ses partisans maîtrisent encore l'appareil du FSN. Ils décident de rejoindre l'opposition et donnent ainsi forme au Parti démocrate Front de Salut National (Partidul Democrat - Front Salvarii Nationale), désignation rapidement raccourcie en Parti démocrate. Les membres fondateurs du FSN qui entendent soutenir le président Iliescu fondent le Front Démocratique de Salut National (Frontul Democratic Salvarii Nationale - FDSN), bientôt transformé en Parti de la Démocratie Sociale Roumaine (Partidul Democratiei Sociale din România PDSR). Dans l'attente des prochaines échéances électorales, un gouvernement d'experts est formé. Une nouvelle Constitution est élaborée qui instaure un régime semi-présidentiel et impose un bicaméralisme égalitaire (le gouvernement est responsable aussi bien devant le Sénat que devant la Chambre des députés). La durée de la législature est accordée avec celle du mandat présidentiel et fixée à quatre ans 1. En septembre 1992, Ion Iliescu remporte l'élection présidentielle avec $61,43 \%$ des voix au second tour et ses partisans obtiennent une majorité relative au Parlement. Nicolae Vacaroiu est appelé à former un nouveau gouvernement. Adversaire affiché de la « thérapie de choc », il fait vœu de défendre les segments les plus fragilisés de la population. S'il sollicite des crédits internationaux, il entend que l'État puisse continuer à contrôler certains secteurs de l'économie. Durant quatre ans, il entretient des relations conflictuelles avec le FMI.

Sa politique est sanctionnée par les électeurs en 1996. La majorité des sièges au Sénat et à la Chambre des députés revient alors aux formations qui prônent une libéralisation accélérée de l'économie : un accord de gouvernement peut ainsi être conclu entre le Parti démocrate de Petre Roman, l'Union Démocratique des Magyars de Roumanie (Uniunea Democrata Maghiara din România UDMR) et la Convention Démocratique de Roumanie (Conventia Democrata Româna - CDR), coalition articulée autour du Parti National Paysan - Chrétien Démocrate (Partidul National Taranesc - Crestin Democrat - PNTCD) et de petites formations libérales. Le représentant de la CDR, Emil Constantinescu, est élu président de la République contre le candidat sortant avec 54,4\% des voix au second tour. Le maire de Bucarest, Victor Ciorbea, est nommé Premier ministre. Membre du PNTCD, il jouit de la faveur des créditeurs internationaux. Il engage rapidement un programme de grandes privatisations mais se heurte à de fortes résistances et est contraint de réviser ses ambitions réformatrices à la baisse. Il s'attire alors les foudres du FMI. Il éprouve corrélativement des difficultés à contrôler sa majorité : son incapacité à conserver la confiance des créditeurs internationaux est interprétée comme un signe d'échec. Au sein de la

1. Une loi électorale vient compéter ce dispositif. Sénateurs et députés sont élus dans des circonscriptions départementales à la représentation proportionnelle par liste avec répartition à la plus forte moyenne. 
coalition au pouvoir, des voix s'élèvent pour réclamer la désignation d'un nouveau Premier ministre. Le Parti démocrate est à la pointe de la contestation ; faute d'obtenir directement satisfaction, il quitte le gouvernement. Ciorbea est poussé à la démission et remplacé par Radu Vasile, ancien porte-parole du PNTCD. Le gouvernement alors formé rencontre les mêmes difficultés que son prédécesseur. Dans un premier temps, il s'attire le soutien des créditeurs internationaux et annonce la mise en œuvre d'un grand plan de réformes. Confronté à la fronde sociale, il doit en rabattre ; ses relations avec le FMI deviennent houleuses et son autorité sur la majorité s'effrite rapidement. En janvier 2000, dix ministres PNTCD présentent leur démission et poussent le Premier ministre - issu de leur propre formation - vers la sortie. Mugur Isarescu, économiste sans réelle envergure politique, est appelé à former un nouveau gouvernement et chargé de gérer les affaires courantes jusqu'aux prochaines échéances électorales.

Depuis 1990, les gouvernements successifs se sont tous efforcés de rester en contact avec les créditeurs internationaux ; ils ont tous veillé à ne jamais dépasser le point de rupture au-delà duquel toute négociation serait devenue impossible et ont tous accepté de libéraliser l'économie roumaine. Leur désaccord n'a porté que sur le rythme et l'intensité des réformes engagées.

Par contraste, trois forces politiques manifestent une opposition de principe à l'économie de marché et préconisent de tourner simplement le dos au FMI 2 : le Parti Socialiste des Travailleurs (Partidul Socialist al Muncitorii - PSM), le Parti de l'Unité Nationale Roumaine (Partidul Unitatii Nationale Române - PUNR) et le Parti de la Grande Roumanie (Partidul România Mare - PRM). Ces partis reposent sur une base militante étroite, n'excédant pas quelques milliers d'individus 3 ; ils n'ont jamais obtenu un succès électoral suffisant pour prétendre exercer seuls le pouvoir. Ils parviennent pourtant à occuper le devant de la scène politique, alimentent le débat public par des discours tonitruants et des propositions maximalistes et se posent en interlocuteurs directs des partis à vocation majoritaire. En multipliant les attaques et les invectives, ils parviennent à les mettre dans l'embarras. En retour, ils sont fréquemment désignés comme les principaux obstacles à la stabilisation de la vie politique roumaine.

Une telle disproportion entre poids nominal et influence effective pose question. Pour l'expliquer, on décrit parfois le PSM, le PUNR et le PRM comme les mauvais génies de la classe politique roumaine : les dirigeants de ces trois partis auraient pour particularité d'exprimer à haute voix des rancœurs et des frustrations partagées secrètement par tous leurs homologues. $N$ 'ayant pas vocation à gouverner, ils n'auraient pas à se soucier de leur image internationale et ne

2. Les partis que nous considérons ici se proclament tous libéraux et revendiquent tous une appartenance au centre. Ces désignations ne résistent pas à la lecture de leurs programmes politiques. Elles participent d'un métalangage politique communément pratiqué dans la Roumanie contemporaine (BOARI, 1994, p. 10).

3. Le PRM revendique aujourd'hui 83392 militants (PRM, 1999d) ; les deux autres formations restent muettes sur leurs effectifs. 
seraient pas contraints de surveiller leur langage ; leurs imprécations rempliraient une fonction cathartique, offrant un exutoire symbolique à des partis à vocation majoritaire corsetés par les mots d'ordre du FMI. Si l'on retient cette optique, on peut estimer avoir affaire à des acteurs secondaires, enivrés par leur propres outrances mais incapables d'élaborer une stratégie politique digne de ce nom.

Formulée de manière aussi fruste, la proposition n'est guère éclairante : elle ne permet pas de caractériser dans leur évolution les relations établies entre les trois formations considérées et les partis à vocation majoritaire.

En retenant les mêmes postulats, il est possible de construire une hypothèse plus élaborée. Une base de réflexion peut être trouvée dans l'étude classique que Georges Lavau a consacrée au Parti Communiste Français. En manière de " binôme métaphorique », il s'agit ainsi d'opposer consulat et tribunat. Le consulat se définit par «l'exercice d'un pouvoir actif », le tribunat peut être décrit comme une «lutte » contre ce pouvoir. Dans la Rome antique, le tribun de la plèbe avait la faculté de bloquer l'application d'une mesure introduite par les consuls. Ces derniers acceptaient que leur pouvoir soit limité de la sorte, voyant dans le dispositif un moyen de canaliser à moindre frais les révoltes plébéiennes. Au terme de négociations avec le tribun de la plèbe et par des chemins détournés, ils parvenaient toujours à imposer leur volonté. En reproduisant ce modèle, le PCF remplit entre 1958 et 1981 une «fonction tribunitienne ». Il ne se comporte pas alors en simple porte-parole de la classe ouvrière mais se pose en représentant de tous les segments marginalisés de la population ; il leur offre « une tribune pour manifester - bruyamment mais légalement - leur hostilité ». Les partis « bourgeois » (les partis « consulaires ») trouvent eux-mêmes avantage à ce positionnement : le PCF figure à leurs yeux un mouvement protestataire intégré au système, qui prévient l'apparition de révoltes populaires plus violentes et moins contrôlables. Encore le parallèle historique ne doit-il pas être poussé trop avant. Le PCF ne dispose pas d'un « pouvoir d'empêcher » comparable à celui de son équivalent antique. Il n'est pas en mesure de bloquer l'application des lois votées par les partis « bourgeois »: «Le tribun avait le pouvoir de veto, il pouvait arrêter le bras des autres magistrats. Le PCF dit "non" mais son "non" n'empêche rien et n'arrête rien ». Sa position d'opposant officiel lui permettant de compter sur un électorat fidèle, il accepte malgré tout de s'y cantonner. Il ne cherche pas à " accéder aux magistratures consulaires, c'est-à-dire au pouvoir actif de faire. Le rôle de tribun lui procure assez de bénéfices de puissance, de force électorale, de prestige. Il ne cherche donc pas réellement à prendre la "relève du pouvoir". Il ne voit que des risques inutiles à dépasser une attitude défensive pour proposer une politique » 4 (Lavau, 1981, pp. 35-36 et 42-46).

4. Nous nous appuyons sur la présentation la plus récente du schéma explicatif. Dans ses premiers écrits, Georges Lavau considérait que le PCF était abusé par les partis «bourgeois » et qu'il remplissait à son insu une « fonction tribunitienne ". La relative pérennité du dispositif examiné l'a amené à réviser son jugement et à considérer que le PCF se complaisait dans son statut. 
Il est tentant de transposer cette analyse au contexte roumain. On pourrait considérer que, par-delà leurs divergences idéologiques, le PDSR, le Parti démocrate, la CDR et l'UDMR exercent une même « magistrature consulaire » et qu'ils s'entendent pour tenir le PSM, le PUNR et le PRM à la marge du système. Il ne s'agirait pas pour eux d'obtenir la disparition de ces derniers mais bien plutôt de leur attribuer une « fonction tribunitienne » : en canalisant les rancœurs et les élans protestataires des populations les plus fragilisées par les réformes économiques, les trois petites formations joueraient un rôle stabilisateur et contribueraient à la pérennité de l'édifice politique constitué. Elles accepteraient sciemment cette position en y voyant une garantie de survie.

Le schéma d'analyse ainsi élaboré peut-il être retenu ? À première vue, il ne manque pas d'attraits, paraissant prêter à une analyse fine des positionnements et repositionnements de chaque parti. Un examen plus approfondi du contexte roumain conduit cependant à mettre sa pertinence en doute : les visées politiques du PSM, du PUNR et du PRM aussi bien que l'attitude des partis à vocation majoritaire semblent marquer un écart avec les définitions proposées par Georges Lavau.

Les petits partis qui retiennent notre attention refusent tout d'abord de se cantonner dans « la lutte ». Ils prétendent ouvertement à l'« exercice d'un pouvoir actif », ne se satisfont pas d'une « fonction tribunitienne » et entendent exercer une « magistrature consulaire ». Ils ne se contentent pas de vitupérer les réformes économiques mais veulent y mettre un terme et remédier aux troubles qu'elles ont déjà provoqués.

Les partis à vocation majoritaire ne cherchent pas tous ensuite à empêcher le PSM, le PUNR et le PRM d'acquérir un « pouvoir actif ». Certains acceptent de s'allicr temporairement avec eux; ils ne les condamnent pas à exercer une « fonction tribunitienne » mais acceptent de les aider à conquérir une « magistrature consulaire ». Ils ne leur demandent pas de canaliser le mécontentement populaire et de prévenir les révoltes incontrôlées mais de participer à la mise en œuvre d'une politique concertée.

Nous devons prendre en compte cette double particularité pour expliquer la disproportion observée entre les maigres ressources dont disposent nos trois formations et le rôle politique majeur qu'elles parviennent à jouer.

Il paraît alors indiqué de recourir à la notion de parti anti-système, définie en 1976 par Giovanni Sartori : un parti anti-système est un parti qui « mine la légitimité du régime contre lequel il se dresse ». En s'appuyant sur un programme cohérent et en proposant une alternative crédible à l'ordre politique institué, il perturbe les équilibres traditionnels, amène les partis à vocation majoritaire à s'entre-déchirer et suscite un appétit de changement chez les électeurs (Sartori, 1976, pp. 130 sq.).

En 1987, Gordon Smith affine et élargit simultanément cette caractérisation. Il l'affine tout d'abord en établissant une sous-distinction entre deux types de par- 
tis anti-système : les partis « légalistes », qui défendent leur projet en s'appuyant sur l'ordre politique établi, et les partis « séditieux », qui se tiennent en marge du jeu politique traditionnel et entendent heurter frontalement le dispositif institué (Smith, 1987, p. 57) 5. Pour élargir la définition de Sartori, Smith suggère ensuite de différencier les ambitions affichées et les résultats obtenus : un parti qui vise à délégitimer l'ordre existant mais qui n'y parvient pas doit pouvoir être qualifié de parti anti-système. Il s'agit également de considérer les relations qu'entretient la formation étudiée avec les forces politiques environnantes : selon que des perspectives de coopération se dessinent ou que tous les horizons paraissent fermés, les responsables d'un parti anti-système adopteront une posture plus ou moins vindicative (Smith, 1987, pp. 58-59).

En étudiant la vie politique roumaine à travers ce prisme, nous pouvons marquer l'originalité du PSM, du PUNR et du PRM. Nous avons affaire à des partis anti-système complexes qui, par leur structuration interne aussi bien que par leurs positionnements externes, mêlent toutes les variables identifiées par Smith.

À des degrés divers, les trois formations tirent leur force d'une structure bifide. Deux factions cohabitent plus ou moins harmonieusement en leur sein. Elles entendent pareillement renverser l'ordre établi et accéder au pouvoir (aucune n'est prête à se satisfaire d'une « fonction tribunitienne »). Elles ne s'opposent que sur des questions de méthode : l'une affiche un profil « légaliste » et l'autre un profil « séditieux ». Ce simple principe de dédoublement doctrinal permet de jouer sur tous les tableaux : le PSM, le PUNR et le PRM peuvent s'efforcer d'intégrer le jeu politique traditionnel et l'attaquer frontalement dans le même temps.

En appui sur cet ordonnancement interne, des négociations heurtées peuvent être menées avec les forces politiques extérieures (les détenteurs d'une « magistrature consulaire » ne cherchent pas à isoler quelques formations susceptibles de canaliser les ardeurs populaires en marge du système). La faction légaliste cherche un terrain d'entente avec les partis à vocation majoritaire qui émettent des critiques mesurées contre les réformes libérales imposées à la Roumanie. La faction séditieuse se dresse concurremment contre ces mêmes partis. Elle fustige leur mollesse et leur dresse un procès en hypocrisie. Elle les place sur le même plan que les formations acquises aux créditeurs internationaux et leur livre une guerre sans merci. Les responsables nationaux qui cherchent à circonvenir le PSM, le PUNR et le PRM se trouvent ainsi placés dans une position des plus inconfortable : il leur faut séduire la faction « légaliste » tout en réduisant au silence la faction « séditieuse ». Dans cette double optique, ils sont contraints de remodeler leurs propres plates-formes politiques. Les trois partis anti-système parviennent donc à exercer sur eux un pouvoir parasitaire.

5. Pour caractériser ces deux types, Gordon Smith emploie les adjectifs radical et revolutionnary. Cette terminologie nous paraît trop connotée et trop restrictive ; elle n'est opérante que dans quelques pays occidentaux et ne prête pas à la généralisation. 


\section{L'ORGANISATION INTERNE DES PARTIS ANTI-SYSTÈME : UN DÉDOU- BLEMENT DOCTRINAL IRRÉGULIER}

Dans chacun des partis anti-système roumains, on trouve une faction légaliste et une faction séditieuse. La faction légaliste affiche un projet cohérent. Elle le présente comme une base de discussion, le soumet à l'appréciation des partis à vocation majoritaire et se montre prête à passer des compromis pour accéder au pouvoir. Elle escompte faire vaciller le système établi en l'attaquant de l'intérieur. Elle est opposée aux participations étrangères dans l'économie roumaine et voue aux gémonies les formations qui en tiennent pour une libéralisation accélérée de l'économie. Elle conteste que les puissances euro-atlantiques et les organismes internationaux puissent jamais apporter à la Roumanie les moyens de son émancipation. Elle considère que les modèles de développement occidentaux ne sont pas adaptés à la société roumaine. En tentant de les lui appliquer à toute force, on ne fait, selon elle, qu'engendrer des structures économiques bâtardes et inefficaces ; partant, on maintient la Roumanie dans une relégation humiliante 6 . La faction légaliste propose une politique alternative, appuyée sur les ressources locales et sur les structures héritées de la période communiste. Elle juge nécessaire de renoncer aux financements internationaux et aux capitaux étrangers pour maintenir ou ramener l'économie nationale dans des formes d'organisation collectives et centralisées. Elle réclame que soient défendues les grandes industries déficitaires. Elle considère que le développement des exportations vers les pays membres de l'OPEP peut remédier aux difficultés structurelles de l'économie nationale. Ces pays ont noué des relations économiques poussées avec la Roumanie dans les années 1970. Ils semblent pouvoir fournir des matières premières et offrir des débouchés à la grande industrie roumaine et présentent en outre l'avantage de ne pas imposer leurs normes économiques à leurs partenaires commerciaux.

Sur le plan interne, la faction légaliste encourage les mouvements de résistance aux réformes. Si elle manifeste quelque sentiment d'hostilité envers la minorité magyare, c'est que cette dernière apporte massivement ses suffrages à l'UDMR, formation qui milite pour une conversion totale à l'économie de marché et bénéficie par ailleurs de puissants relais dans les organisations internationales honnies.

Aux côtés de la faction légaliste, une faction séditieuse donne le plus généralement de la voix. Elle se contente d'imputer les difficultés que connaît la Roumanie à des agents extérieurs et multiplie les attaques contre l'ordre poli-

6. Par un singulier court-circuit historique, on peut trouver là un écho à la fameuse théorie des « formes sans fond », développée dans les années 1860 par le mouvement conservateur Junimea (La jeunesse). Selon cette théorie, les formes institutionnelles occidentales sont importées et artificiellement posées sur le sol roumain. Dès lors que le substrat organique de la nation ne leur offre pas un fond idoine, elles sont source de perturbations. 
tique institué, supposé favoriser ces agents. Ses dirigeants sont avant tout des imprécateurs. Ils vilipendent le capitalisme bisnitar - qualificatif roumain dérivé de l'anglais business, qui évoque à la fois le petit commerce de bazar et le trafic irrégulier. Ils sont hostiles aux minorités nationales, principales instigatrices et principales bénéficiaires à leurs yeux dudit capitalisme. Les Tziganes sont leur cible désignée : ils sont accusés de se livrer à la spéculation et de stocker les produits pour en faire monter les prix, sont jugés responsables des pénuries qui frappent régulièrement les marchés urbains et sont regardés comme les principaux corrupteurs de l'administration.

La minorité magyare est attaquée sur un registre sensiblement différent. Elle est décrite comme l'agent actif d'un complot contre la Roumanie ourdi par les États-Unis et la Russie. Les lobbies hongrois au Congrès américain et au Conseil de l'Europe ou la Fondation Soros sont considérés comme des courroies de transmission. La « finance juive internationale » est parfois caractérisée comme l'inspiratrice suprême de la machination. Bien que les Juifs ne soient plus qu'au nombre de 8955 en Roumanie (selon le recensement de 1992), on voit ainsi affleurer des discours antisémites (Sonescu, 1998, p. 11).

Faction légaliste et faction séditieuse affichent des orientations doctrinales bien différenciées. Pour autant, elles ne sont pas hermétiquement fermées l'une à l'autre. Dans des rapports de force variables et sur un mode plus ou moins frictionnel, elles cohabitent au sein des partis anti-système. Tout en affichant une façade unitaire, le PSM, le PUNR et le PRM peuvent se positionner sur plusieurs registres : ils fulminent contre les partis à vocation majoritaire mais restent ouverts à toute proposition d'alliance. Si l'équilibre entre les deux options n'est pas automatique, chaque parti est susceptible de s'en approcher à un stade ou à un autre de son évolution 7 .

Créé en 1992, le Parti Socialiste du travail (PSM) est très largement dominé par une faction légaliste 8 . Il est considéré le plus souvent comme l'héritier direct du Parti Communiste Roumain. Il est dirigé par Ilie Verdet, Premier ministre de Ceausescu entre 1979 et 1982. Dans sa revue mensuelle Socialistul (Le Socialiste) et dans son quotidien Vremea (Le Temps), il exalte les réalisations de la période communiste et ne récuse que l'orientation adoptée par le régime à la

7. Michael Shafir définit les partis que nous étudions ici comme des « partis de la conservation intégrale » (parties of radical continuity), attachés à préserver les structures héritées de la période communiste. Il les oppose aux «partis de la restauration intégrale » (parties of radical return), désireux de faire renaître la geste fasciste de l'entre-deux-guerres (SHAFIR, 1994, pp. 71-89). Cette présentation occulte les affrontements observés à l'intérieur des partis dits de la conservation intégrale. La symétrie sur laquelle elle repose nous paraît quelque peu forcée par ailleurs. Les partis dits de la restauration intégrale organisent des défilés et publient des revues antisémites, mais ils n'ont aucun poids politique (ils recueillent moins de $0,1 \%$ des voix en 1992). Ils ne présentent guère qu'un caractère folklorique. Ils participent d' « un spiritualisme snob, où se découvre bien plus une esthétique qu'une mystique, rendu hystérique par sa propre inauthenticité » (ANTOHI, 1998, p. 131).

8. En 1992, le PSM succède à une formation dénommée Union de la Gauche Démocratique. 
fin des années 1980. Il se réclame de l'École de Fribourg, soutenant que le modèle marxiste continue de fournir des réponses pertinentes aux problèmes roumains. Il explique que les réformes introduites depuis 1990 sont à l'origine « d'un écocide, d'un zoocide et d'un génocide » - ce dernier terme devant caractériser les pénuries qui frappent le peuple roumain. Le PSM prend pour cible les créditeurs internationaux, mais il s'attaque aussi à leurs « suppôts roumains ». Il préconise un maintien des structures collectives et demande un renforcement du contrôle sur la production et sur la distribution, seule façon selon lui d'assurer la sécurité alimentaire de la Roumanie (PSM, 1992, p. 3 ; 1999, p. 2).

La faction séditieuse est faible et bien muselée. Un clivage est pourtant observé au sein du parti. Verdet et son entourage pâtissent d'une image Ancien Régime. Rangée derrière Tudor Mohora, une partie des militants attend que le PSM se débarrasse de ce stigmate. Elle considère que le projet anti-système ne pourra être efficacement défendu tant que les dirigeants ne marqueront pas clairement leur rupture avec la période communiste. Elle demande que soient abandonnées les références à l'œuvre de Ceausescu et que le programme développé soit plus explicitement tourné vers l'avenir. En somme, l'affrontement entre direction et base militante porte moins sur les principes (la même ligne légaliste est défendue dans les deux cas) que sur la forme. Les débats sont souvent confus dans la mesure où la faction séditieuse minoritaire, désireuse de renverser la direction, tend à soutenir les éléments contestataires de la faction légaliste.

Au regard de ses prétentions initiales, le PSM obtient en 1992 des résultats électoraux plus qu'honorables. Il ne conquiert que 11 mairies aux élections locales mais obtient 13 sièges à la Chambre des députés (3,04\% des suffrages exprimés) et 5 au Sénat $(3,19 \%)$. Ces résultats renforcent la direction du parti et font taire momentanément les critiques internes. En mars 1993 néanmoins, une «branche jeune » fait scission dans le judet (département) du Gorj. En 1995, les contestataires lancent une plus vaste offensive : sept responsables du PSM, dont quatre parlementaires, donnent leur démission, fondent le Parti Socialiste (Partidul Socialist - PS) et portent Mohora à leur tête. Ils dénoncent les « orientations staliniennes » de Verdet et de ses compagnons mais conservent une position fermement anti-système. Ils s'insurgent toujours contre les « diktats » du FMI. S'opposant aux privatisations, ils les décrivent comme un « transvasement illégal de la propriété publique dans la propriété privée ». Ils réclament que soit maintenu et généralisé le contrôle de l'État sur les prix. Pour cohérentes que soient ces vues, elles s'accompagnent de choix politiques assez incongrus. Soucieux de se démarquer du PSM, Mohora en vient à afficher des signes de bienveillance envers... les partis qui s'attachent à promouvoir les réformes libérales. Cette tactique est logiquement contestée par la base et provoque de nouvelles fractures : le vice-président du PS, Gheorghe Cazan, démissionne en criant à la trahison du programme anti-système soutenu par les militants. Il est suivi par 50 dirigeants du parti (Ivan, 1993, p. 6 ; PSM, 1996, p. 1).

Ces défections en chaîne sont source d'affaiblissement. Aux élections législatives de 1996, le PS recueille 2,3\% des suffrages exprimés (Chambre des dépu- 
tés et Sénat), le PSM en obtient 2,15\% (Chambre des députés) et 2,16\% (Sénat). Ni l'une ni l'autre formation ne dépasse le seuil de $3 \%$ en deçà duquel aucune représentation parlementaire ne peut être accordée. Quelques dirigeants sont toutefois parvenus à s'implanter localement, de sorte que des résultats plus flatteurs sont obtenus aux élections municipales. Le PSM, qui détenait déjà 11 mairies, en conquiert encore 109, parmi lesquelles trois préfectures : Craiova, Giurgiu et Braila. Le PS remporte 43 mandats de maires, essentiellement dans des petites communes rurales.

Après avoir goûté les joies et apprécié les limites de l'isolement, Mohora et ses compagnons cherchent à se rapprocher du PSM. Au début de l'année 1999, ils proposent la reconstitution d'un parti unitaire. Les négociations trainent en longueur. Le PS réclame une distribution paritaire des sièges à l'intérieur des nouvelles instances dirigeantes (Bureau exécutif et Comité directeur); il appuie sa revendication sur les résultats obtenus aux élections nationales. Verdet objecte que sa formation détient un nombre supérieur de mandats locaux et qu'elle doit être représentée à proportion. Dans l'impossibilité de trouver un compromis, les deux formations renoncent finalement à se ressouder (PSM, 1997/2-4).

Le Parti de l'Unité Nationale Roumaine (PUNR) est plus nettement partagé que le PSM entre faction séditieuse et faction légaliste. La première domine tout d'abord, mais elle est progressivement supplantée par la seconde.

Un Parti de l'Unité Nationale Roumaine de Transylvanie (Partidul Unitatii Nationale Române din Transilvania-PUNRT) est fondé à Brasov en mars 1990. Il présente un caractère régionaliste et est tourné prioritairement contre l'UDMR. Il peut être défini comme la branche politique de Vatra Româneasca (L'âtre roumain). Cette « association culturelle », dirigée par Radu Ceonta, est l'organisatrice des manifestations anti-magyares qui ont ensanglanté la ville de Tîrgu Mures en 1990. Elle se propose de « freiner les efforts engagés pour libéraliser les relations européennes, en les sapant si nécessaire, cela pour la raison qu'elles peuvent avoir une influence néfaste sur l'âme authentique et traditionnelle du peuple roumain et favoriser l'érosion du noble et puissant sentiment national roumain » (Deletant, 1991, pp. 40-44; Gallagher, 1992 et 1997).

Le PUNRT est dirigé successivement par M. Ivasiuc et Al. I. Crisan. Au début de l'année 1990, en vue des élections législatives, il se coalise avec un groupuscule nommé Parti Républicain (Partidul Republican). Une Alliance pour l'Unité de la Roumanie (Alienta pentru Unitatii României) est ainsi formée. Elle recueille $2,12 \%$ des suffrages exprimés et obtient 9 sièges à la Chambre des députés. Son assise est encore limitée à la Transylvanie et, de façon plus restrictive encore, aux judete de Mures et de Cluj où ses candidats obtiennent respectivement $34,36 \%$ et $13,5 \%$ des voix.

En novembre 1990, le PUNRT fusionne avec une micro-formation moldave. Il peut ainsi se débarrasser de son étiquette régionaliste et espérer sortir de sa marginalité. Il ouvre une antenne à Iasi et se transforme en PUNR. Radu Ceonta prend sa direction. Dès ce moment, une division est observée entre faction sédi- 
tieuse et faction légaliste. La faction séditieuse tient l'appareil du parti : elle est contrôlée par Ceonta lui-même. Elle s'efforce de canaliser dans des diatribes anti-magyares les tensions provoquées par les réformes libérales. Elle entend renverser l'ordre politique établi pour mettre un terme aux privatisations « antidémocratiques, antipopulaires et antinationales ». Elle trouve une audience parmi les populations âgées et dispose aussi d'importants soutiens dans l'armée.

La faction légaliste est animée par Valeriu Tabara et Ioan Gavra. Elle réclame une suspension des réformes économiques et le simple perfectionnement de l'économie dirigée. Elle ne porte qu'un intérêt secondaire à la question magyare. Elle conteste l'orientation donnée au PUNR par Ceonta et souhaite collaborer avec les partis à vocation majoritaire qui se montrent les plus critiques envers les réformes libérales.

Aux élections municipales de février 1992, le PUNR remporte 96 mairies. Il s'implante particulièrement dans les judete de Bihor, Maramures, BistritaNasaud, Alba (Pepine, 1994, pp. 4-5). Les élections nationales doivent se tenir sept mois plus tard. Les deux factions rivales s'affrontent pour la désignation du chef de campagne et du candidat national. Le nouveau maire de Cluj, Gheorghe Funar, s'impose comme personnalité de compromis. Diplômé en « gestion des fermes collectives », il passe alors pour un « modéré », attaché à élargir l'assise du parti à la Roumanie tout entière. Il semble vouloir élaborer un programme économique cohérent. Dans un discours retentissant, il critique Ceonta pour son approche « provinciale » et son orientation «droitière ». Dans le même temps, il dénonce à l'envi le capitalisme bisnitar et la conspiration des bozgori (terme péjoratif qui désigne les Magyars). Sous sa férule, le PUNR remporte un franc succès aux élections législatives : il obtient 30 sièges à la Chambre des députés (avec un score de 7,71\%) et 14 au Sénat (avec un résultat de 8,12\%). En Transylvanie, il recueille un quart des suffrages exprimés. À l'élection présidentielle, Gheorghe Funar obtient $10,87 \%$ des voix. Porté par ces chiffres flatteurs, il prend la direction du PUNR. Il renonce alors à sa posture intermédiaire et penche résolument dans le sens de la faction séditieuse. Il évoque un complot international contre les intérêts roumains. Dans sa ville, il s'illustre régulièrement par des mesures symboliques : après avoir interdit les inscriptions en langue magyare, il décide de faire graver sur le socle de la statue de Matthias (roi de Hongrie entre 1458 et 1490, né à Cluj) une citation de l'historien Nicolae Iorga évoquant la victoire du prince Etienne le Grand (Stefan cel Mare) sur les armées magyares. Devant la levée de protestations, il menace de faire fondre ladite statue et d'ériger à sa place un monument à la mémoire du héros national roumain Avram Iancu. Il s'oppose enfin à la tenue d'une conférence sur « le gouvernement local autonome » organisée à Cluj par une association néerlandaise.

Si la faction légaliste voit la direction du parti lui échapper, elle n'est pas totalement écrasée - elle contrôle les élus du PUNR. Ioan Gavra préside le groupe PUNR à la Chambre des députés. Il conteste régulièrement les initiatives de Funar et recherche une entente avec les grands partis qui critiquent avec mesure la soumission de la Roumanie aux mots d'ordre des créditeurs internationaux. En 
août 1993, un Congrès du PUNR est organisé à Cluj. La motion de Funar est rejetée. Sans pouvoir déloger encore le président du parti, la faction légaliste monte en puissance.

En juin 1996, le PUNR s'impose à la tête de 147 municipalités. Il progresse essentiellement dans les campagnes et perd le contrôle des plus grandes villes, à l'exception notable de Cluj. Aux élections législatives de novembre 1996, il ne recueille que $4,36 \%$ et $4,22 \%$ des suffrages exprimés. Sa représentation passe de 30 à 18 sièges à la Chambre des députés et de 14 à 7 sièges au Sénat, les députés et sénateurs récusés par les électeurs appartenant majoritairement à la faction séditieuse. En dépit de fortes contestations internes, Gheorghe Funar parvient à imposer sa candidature à l'élection présidentielle. En 1992, il avait rassemblé $10,87 \%$ des voix mais n'en recueille plus que 3,22\%. Il appelle ses électeurs à reporter leurs voix au second tour sur... Emil Constantinescu. Entendant ainsi sanctionner le président sortant, accusé d'avoir trahi la Roumanie en cherchant une entente avec la Hongrie, il se trouve par le fait soutenir le premier partisan des réformes libérales, qui plus est directement allié à l'UDMR. La faction séditieuse sort discréditée du scrutin.

En février 1997, Funar est poursuivi pour abus de biens sociaux (il est accusé d'avoir utilisé le téléphone et la télécopie de la mairie de Cluj pour des communications privées très onéreuses) ${ }^{9}$. Le prétexte est ainsi fourni qui permet à l'appareil dirigeant de le renverser. La ligne politique qui a conduit le parti à la déroute électorale est sanctionnée. La faction légaliste prend les commandes. Fidèle à Funar, la section de Cluj exclut en représailles - et tout temporairement - l'un des principaux artisans du changement d'orientation, Ion Gavra. En mars, une autre figure légaliste, Valeriu Tabara, est portée à la tête du PUNR.

Le Parti de la Grande Roumanie (PRM) passe lui aussi d'une orientation séditieuse à une orientation légaliste, mais de façon moins brutale et moins tranchée. À sa fondation, en octobre 1991, il est contrôlé presque entièrement par une faction séditieuse ; il entend drainer le lectorat de l'hebdomadaire populiste et xénophobe România Mare (La Grande Roumanie), publié à 500000 exemplaires à partir de 1990 et complété à partir de 1992 par Politica (La politique). Dirigé par Corneliu Vadim Tudor, ancien poète de cour, il se ferme à toute alliance et pré-

9. Funar s'est auparavant compromis dans l'affaire Caritas, affaire très représentative du capitalisme bisnitar qu'il dénonce par ailleurs. En 1991, la " société d'aide mutuelle » Caritas commence à drainer l'épargne des citoyens de Brasov ; appuyée sur un système pyramidal, elle promet huit fois la mise initiale à ses adhérents. Chaque étage de la pyramide récupère la somme apportée par l'étage supérieur. En juin 1992, la société étend ses activités à la ville de Cluj. Son responsable, Ion Stoica, offre de fortes sommes d'argent à la mairie, dirigée par Funar, pour financer la construction de monuments à la gloire des héros nationaux. Il présente par ailleurs le système Caritas comme un moyen « d'aider les Roumains de Transylvanie à devenir plus riches que les Hongrois ». Le président du PUNR lui apporte sa bénédiction. Un cinquième de la population roumaine engloutit ses économies dans la pyramide financière (un milliard de dollars au total, soit la moitié du budget de l'État). L'édifice s'écroule sur luimême en 1993 (MıHU, 1994, pp. 107-120). 
tend réhabiliter la politique de Ceausescu. Le Conducator a, selon lui, « apporté plus de bien que de mal » à la Roumanie : il lui a permis d'échapper à l'emprise des créditeurs internationaux, l'a dotée d'un « potentiel économique relativement élevé », a fait en sorte que les Roumains ne soient pas « terrorisés par les Tziganes et les Magyars ». Sa chute est interprétée comme le résultat d'une manœuvre russe et hongroise. Le PRM dissocie la personne de Ceausescu de l'idéologie communiste, « introduite en Roumanie par des Juifs, des Hongrois, des Russes et des Tziganes » (par son imprécision, cette dernière catégorie permet de regrouper tous les individus qui ne rentrent dans aucune autre). Les communistes ont jeté dans les prisons des milliers de «Roumains authentiques » 10 . Après que Ceausescu a été lâchement exécuté, la « réforme criminelle » a mis la Roumanie sous la coupe des banques étrangères. L'économie roumaine est parallèlement « colonisée » par les puissances occidentales (Stefan-Scalat, 1999, p. 9 ; Voicu, 1993a, pp. 4-5 et 20-21 et 1993b, p. 6 ; Neumann, 1993, p. 12). Des « forces occultes », « soutenues de l'extérieur et manipulées depuis l'étranger », œuvrent au « démembrement de la Roumanie ». Le PRM dénonce « l'hystérie qui pousse à privatiser la richesse nationale à tout prix ». Il qualifie le FMI et la Banque mondiale $d$ ' « organisations mafieuses ». Il propose la création d'un «Comité national d'investigation sur les activités anti-roumaines », chargé de dévoiler et de juger les « actes de trahison nationale, de déstabilisation et de sabotage commis par des Roumains ou par des étrangers ». Il attend que les ministres et fonctionnaires « impliqués » dans la politique de privatisation soient condamnés pour « haute trahison ». Il demande que soit instauré un contrôle sur les mouvements de population, seul moyen d' « empêcher l'infiltration de l'État roumain par des éléments étrangers hostiles à ses intérêts ». Tudor réclame de façon répétée la formation d'un gouvernement militaire qui, dans un délai de deux ans, pourrait rétablir l'ordre et déjouer le «complot international » ourdi contre la Roumanie (PRM, 1993, pp. 6-8 ; 1996b, pp. 10-12) 11.

Ce discours séditieux permet au PRM d'obtenir 13 sièges à la Chambre des députés en 1992 (3,9\% des suffrages exprimés) et 6 au Sénat (3,85\%). Les candidats du parti connaissent plus de difficultés à s'imposer dans les scrutins locaux, trois d'entre eux seulement accédant à la fonction de maire. Bien que toujours minoritaire au sein du parti, la faction légaliste commence alors à s'ex-

10. Tudor se flatte d'avoir démissionné du PCR en juin 1989 (!) en signe de protestation contre la destruction d'un bâtiment où avait séjourné le poète national Mihai Eminescu.

11. La thématique du complot et le discours xénophobe ainsi développés ont conduit Tudor à nouer des liens (des « relations appropriées » selon ses propres termes) avec le Front National français (il assiste au Congrès du FN à Strasbourg en 1996 ; Jean-Marie Le Pen est l'invité d'honneur du Congrès du PRM la même année). Ce rapprochement ne permet pas de caractériser le PRM comme un parti d' " extrême droite » - raccourci que s'autorise notamment Michael Shafir (SHAFIR, 1993, p. $160 ; 1994 ; 1999$ ). Il nous paraît imputable à une interférence entre repères politiques occidentaux et réalités roumaines. Nous noterons ainsi qu'en usant du métalangage évoqué plus haut, le PRM se présente lui-même comme un parti de « centre-gauche », sans voir là aucune contradiction avec ses sympathies lepénistes affichées. 
primer. Elle propose que soit dépassé le stade de la simple imprécation et que soit formulé un programme constructif, susceptible d'apporter une réponse cohérente aux difficultés rencontrées par la population roumaine. Elle entend par ailleurs nouer des alliances avec les dirigeants qui, membres d'autres formations, partagent certaines de ses préoccupations. En novembre 1992, Radu Theodoru démissionne du PRM. Il dénonce les orientations antisémites et xénophobes du parti, imputables selon lui à un groupe de dirigeants déconnectés de la réalité et incapables de comprendre les aspirations de la base. Il est suivi par plusieurs fédérations départementales et par quelques centaines de militants. Au début de l'année 1993, Ion Hristu s'écarte de Tudor pour des motifs semblables ; Constantin Moldovan et Victor Stoiescu lui emboîtent le pas en février 1994.

En 1996, à l'approche des élections, la faction séditieuse prend seule les rênes du parti. Elle collige toute ses propositions dans une Doctrine nationale. Elle parvient ainsi à rassembler la majorité des votes protestataires et à empiéter sur l'électorat traditionnel des formations concurrentes. Aux élections locales du mois de juin, le PRM remporte 57 mairies, chiffre modeste mais qui marque une progression certaine. Aux élections législatives, 19 candidats du PRM sont élus à la Chambre des députés $(4,46 \%$ des suffrages exprimés) et 8 au Sénat $(4,54 \%)$. Au premier tour de l'élection présidentielle, Tudor recueille $4,72 \%$ des voix.

Le PRM dépasse alors les partis de Verdet et de Funar. À l'inverse du phénomène observé au sein du PUNR, c'est ce succès relatif qui provoque un basculement vers l'option légaliste. Les deux autres formations anti-système sortant déstabilisées du scrutin, le PRM voit s'ouvrir à lui un nouvel espace. Il commence à nourrir des ambitions plus affirmées, entendant s'imposer comme le principal parti de l'opposition. Il doit pour cela élargir sa plate-forme : il n'est plus tant question de mettre au pilori les Tziganes et les Magyars que d'opposer un projet politique crédible au programme appliqué par le gouvernement. Il ne s'agit plus en bref de se complaire dans une xénophobie flamboyante et d'attaquer les institutions qui servent les « intérêts de l'étranger » mais de pénétrer l'ordre politique établi pour l'attaquer de l'intérieur (PRM, 1996b). Des « améliorations » sont ainsi apportées à la Doctrine nationale qui mettent l'accent sur une exigence de « justice sociale ». Par un Programme de gouvernement, le parti entend promouvoir un «nationalisme éclairé ». Il commence à formuler des propositions économiques précises, cherchant à démontrer sa « capacité à accéder au pouvoir et à remettre la société roumaine sur le chemin du développement et de la modernisation, de la prospérité et de la dignité ». Il se donne pour « objectif stratégique » de « protéger et développer les ressources, virtualités et potentialités de la nation roumaine de façon à tirer les bénéfices de la modernité et de la prospérité ». Pour défendre « les intérêts vitaux du peuple roumain », il préconise la « planification macroéconomique », le contrôle absolu de l'État sur les prix et « le rétablissement d'un équilibre entre l'offre et la demande ». Il attend que soit « soutenu, consolidé et développé le capital autochtone ». Il réclame un « renforcement du contrôle de l'État sur les entreprises publiques qui présentent une importance stratégique ». Il entend que soient «protégées les ressources 
nationales » et tient que « la privatisation et la restructuration de l'économie nationale doivent s'opérer non par la liquidation et la destruction mais de façon rationnelle et sous un contrôle rigoureux, dans l'intérêt du Peuple roumain ». Pour développer le secteur agricole, il propose une «consolidation des formes d'association et de coopération $»$. Il refuse d'envisager une nouvelle redistribution des terres et un démantèlement des fermes d'État (PRM, 1997a, pp. 9-10 ; 1997c, pp. 5-7; 1999c, p. 1).

Les mêmes dirigeants qui défendaient hier une ligne rigoureusement séditieuse adoptent désormais une orientation légaliste - et ce « changement dans la continuité » à la tête du parti marque un autre écart avec l'évolution du PUNR. Notons bien que le discours séditieux n'est pas abandonné ; il est simplement recouvert par la plate-forme légaliste et refait surface dès que la conjoncture s'y prête. Tudor manie toujours l'outrance : si le PRM courtise un nouvel électorat, il n'est pas question pour lui d'abandonner l'ancien. Entre la dénonciation du capitalisme bisnitar et la défense d'un projet économique dirigiste, les modulations sont somme toute aisées 12. Exclu du PUNR selon les modalités exposées plus haut, Funar intègre le PRM en novembre 1998 et s'y voit attribuer le rang de Secrétaire général. Aux côtés de Tudor, il prend en charge la défense des options séditieuses.

Dans la même optique de recadrage a minima, le PRM passe alliance avec ses homologues anti-système, tous appuyés désormais sur un programme légaliste. Pour permettre au PSM de survivre à son échec électoral, il s'engage à « porter sa voix » au Parlement. En février 1998, le PRM et le PUNR signent un « protocole de collaboration » : une Grande Alliance pour la Renaissance de la Patrie (Mare Alianta pentru Renasterea Patriei) est constituée qui doit permettre aux deux formations de mener des « actions communes » au Parlement et de s'entraider « sur un plan logistique » (PRM, 1997b, p. 2).

En diversifiant leurs approches, les dirigeants du parti gagnent de nouvelles recrues. Miron Cozma adhère notamment au PRM. Leader syndical et chef charismatique des mineurs de la vallée du Jiu, orchestrateur général des grèves et manifestations de « gueules noires », il a été condamné en 1996 pour « atteinte à la sûreté de l'État ». Libéré en août 1998, après un an et demi de captivité, il se pose en grand idéologue et en premier adversaire de la politique de libéralisation. Il tient une conférence de presse durant laquelle il explique avoir beaucoup

12. L'historien Viorel Roman apporte son soutien à Tudor. Il publie régulièrement des lettres ouvertes dans Romania Mare et Politica. Il offre ainsi une caution intellectuelle au PRM. Son parcours est des plus intéressant. Dans des travaux anciens, inspirés par les thèses d'Immanuel Wallerstein, il s'est efforcé de démontrer que les puissances capitalistes occidentales ont continûment exploité les provinces roumaines jusqu'en 1945. Il prolonge aujourd'hui cette lecture en décrivant les créditeurs internationaux comme les fomentateurs d'une nouvelle entreprise d'asservissement. La même trajectoire est suivie par Ilie Badescu, sociologue hétérodoxe et disciple affiché de Wallerstein à la fin des années 1980, devenu président d'honneur d'un groupuscule néo-fasciste et toujours attaché à fustiger l'impérialisme occidental. 
lu en prison et s'être forgé une doctrine politique. Il se propose de la défendre aux côtés de Tudor. Quelques remous politico-judiciaires sur lesquels nous reviendrons l'empêchent d'aller bien loin en ce sens. L'ouverture du parti à ses propositions n'en est pas moins significative.

On voit que le dédoublement doctrinal des partis anti-système prend des formes variées et évolutives. Il est plus ou moins poussé. Ici, il est facteur de tensions, là, il prête à des remodelages en douceur. Dans tous les cas, il permet de jouer sur des registres dissociés. Les trois formations entendent pénétrer l'ordre politique institué et le miner de l'intérieur, mais ne renoncent pas pour autant à l'attaquer frontalement. En s'appuyant sur les deux facettes de leur doctrine, elles peuvent envisager une collaboration avec des formations à vocation majoritaire et se présenter simultanément comme les adversaires intraitables du système institué. Les équilibres ainsi obtenus sont précaires et évolutifs. Lorsque la conjoncture politique est favorable, ils permettent aux partis anti-système de subsumer leur faible poids électoral et de jouer un rôle central dans la vie politique roumaine. Le PSM, le PUNR et le PRM ne sont pas contraints de renoncer à l'« exercice d'un pouvoir actif» et de se replier sur une « fonction tribunitienne ». Ils peuvent se contenter d'entretenir des relations houleuses avec les partis à vocation majoritaire qui s'opposent à une libéralisation rapide de l'économie roumaine. En se maintenant à la limite du point de rupture, et nonobstant quelques erreurs de réglage, ils exercent sur eux un pouvoir parasitaire des plus efficaces.

\section{L'INFLUENCE DES PARTIS ANTI-SYSTÈME SUR LA VIE POLITIQUE ROUMAINE : UN POUVOIR PARASITAIRE ÉPROUVÉ}

Dans les premières années qui succèdent à la chute de Ceausescu, le contexte politique n'est pas favorable aux partis anti-système ; il ne leur offre aucun point d'appui 13. Le FSN est attaché à redorer son blason diplomatique et ne peut envisager de conclure une alliance avec des formations qui s'attaquent directement aux organisations internationales. Sur le plan interne, ses succès électoraux lui assurent du reste une large assise politique et les pressions des factions séditieuses ne peuvent le déstabiliser. Si la politique menée par le gouvernement provoque quelques tensions, c'est à l'intérieur même du parti au pouvoir que les critiques les plus virulentes se font entendre.

En 1992, l'équilibre politique devient plus chancelant. Ion Iliescu remporte alors l'élection présidentielle, mais les élections législatives lui sont moins favorables. S'il dépasse les autres partis, le FDSN ne dispose pas d'une majorité absolue : il n'obtient que 117 sièges sur 343 à la Chambre des députés et 49 sur

13. Les partis anti-système ne sont pas immédiatement identifiés. Ainsi que nous l'avons noté, le PUNR est créé en 1990, le PRM en 1991 et le PSM en 1992. Dans chacun des cas cependant, des associations ou des pôles de réflexion sont constitués qui préfigurent les rassemblements à venir. 
143 au sénat. Le Parti démocrate refuse de lui apporter son soutien. De part et d'autre du pôle constitué autour du président, deux blocs symétriques se forment alors. Un premier bloc regroupe, sans les souder formellement, les formations favorables à une libéralisation accélérée de l'économie (UDMR, Parti démocrate, CDR). Il détient 152 mandats à la Chambre des députés (27 reviennent à l'UDMR, 43 au Parti démocrate et 82 à la CDR) et 64 au Sénat (34, 12 et 18). Un bloc anti-système est composé par le PUNR, le PRM et le PSM. Il rassemble 59 députés (respectivement 30,16 et 13) et 25 sénateurs $(14,6$ et 5 ).

Un petit parti charnière, le Parti Démocrate Agrarien de Roumanie (Partidul Democrat Agrar din România - PDAR), dispose de 5 sièges au Sénat. De nature étroitement catégorielle, il défend les intérêts des anciens chefs de coopérative attentifs à tirer profit du passage à une agriculture de marché. En échange de quelques maroquins, il accepte dans un premier temps de servir d'appoint au PDSR : son soutien permet de composer une majorité et de constituer un gouvernement homogène sous la férule de Nicolae Vacaroiu.

En juin 1994, trois élus du PDAR cessent de soutenir le gouvernement. En l'absence d'une majorité absolue, cette petite défection suffit à inverser le rapport de force parlementaire : une motion de censure étant déposée, la majorité en place est arithmétiquement menacée. L'opposition libérale compte au total 216 élus (Chambre des députés et Sénat confondus). Les trois représentants du PDAR qui ont viré de bord entreprennent de se concilier les 44 parlementaires du PUNR. Le PDSR dispose de 166 voix, auxquelles ne peuvent s'ajouter que les 40 voix du PRM et du PSM et les deux voix restantes du PDAR (les minorités nationales disposent de 15 représentants qui s'abstiennent en général lorsque la question à l'ordre du jour ne les concerne pas directement). Qu'une coalition de circonstance se forme entre les partis d'orientation libérale, le PUNR et les trois renégats du PDAR (ces derniers servant en quelque sorte de tampon), et la motion de censure est adoptée (263 voix sur 486 ). Les tractations sont nombreuses durant les trois jours qui précèdent le vote. Les dirigeants du PUNR, courtisés de toute part, refusent finalement de faire choir le gouvernement. Transformés en pompiers de la majorité, il escomptent obtenir bientôt quelque récompense.

Le mois suivant, les partis de l'opposition réclament la suspension du président Iliescu. Ils l'accusent d'avoir porté «atteinte à l'indépendance de la justice » dans quelque affaire politico-financière et d'avoir par conséquent violé la Constitution. La procédure compte quatre étapes : elle doit être engagée par un tiers au moins des députés et sénateurs ; elle doit ensuite obtenir l'aval de la Cour constitutionnelle ; elle doit encore recueillir la majorité des votes dans les deux chambres ; elle doit enfin être approuvée par référendum dans un délai de 30 jours. Seules les deux premières étapes sont franchies dans le cas qui nous occupe. De nouveau, le PUNR reste fidèle à la majorité constituée : après avoir sauvé le gouvernement, il sauve le président de la République. Il est alors en position de force ; monnayant logiquement son soutien, il pousse le Premier ministre à suivre une politique plus résolument anti-système. Le PRM et le PSM 
sont inspirés par son exemple : ils comprennent qu'ils peuvent faire chanter le gouvernement et s'y exercent sans retenue. Le dédoublement doctrinal révèle alors toute son utilité : la faction séditieuse peut attaquer le parti au pouvoir et le soumettre à forte pression, la faction légaliste peut, dans le même temps, se montrer plus conciliante et négocier avec lui un accord politique.

Les dirigeants en place sont conscients du danger que recèle une alliance ouverte avec les partis anti-système. Ils savent que leurs interlocuteurs internationaux condamnent cette perspective et qu'ils sont prêts à refuser toute aide à la Roumanie dans l'hypothèse où elle serait retenue. Sur le plan interne, les résistances ne sont pas moindres : au sein du PDSR, une faction commence de se constituer derrière le ministre des Affaires étrangères, Teodor Melescanu. Elle entend maintenir le parti sur une ligne modérée et se montre hostile à toute compromission avec Funar, Tudor ou Verdet.

Le Premier ministre est pris entre deux feux. Il cherche donc à faire en sorte que le PUNR, le PRM et le PSM continuent aussi longtemps que possible de lui apporter un soutien sans participation. Pour calmer leur appétit croissant, il ne peut plus se contenter de donner une vague coloration anti-système à sa potion politique mais doit introduire des mesures roboratives.

Un remaniement ministériel lui permet d'intégrer au gouvernement deux personnalités proches des partis anti-système, présentées comme «indépendantes » aux créditeurs internationaux. Le nouveau ministre de la Justice, Iosif Gavril Chiuzbaian est un ami de Tudor et un compagnon de route du PUNR ; désigné ministre des Transports, Aurel Novac gravite également dans l'orbite de Funar. Il est à noter par ailleurs que le général Nicolae Spiroiu, jusqu'alors ministre de la Défense, n'est pas reconduit dans ses fonctions. Le PRM réclamait sa démission depuis plusieurs mois, l'accusant de chercher à démanteler l'industrie roumaine d'armement à des fins d'enrichissement personnel.

Ces ouvertures ne suffisent pas à rassasier les partis anti-système. Le PUNR ne se contente plus de simples aubades. Il entend pousser son avantage. En août 1994, il réclame d'entrer au gouvernement par la grande porte. Il annonce qu'en cas de refus, il ralliera l'opposition. Le Premier ministre obtempère mais veille à ne solliciter que des personnalités légalistes : Valeriu Tabara est désigné ministre de l'Agriculture et Ioan Ovidiu Muntean ministre de la Communication. Chiuzbaian et Novac restent à leurs postes et officialisent leur adhésion au PUNR. La formation de Funar détient dès ce moment quatre postes ministériels. Dans les mois qui suivent, cinq membres du PUNR accèdent à la fonction de préfet et les militants du parti intègrent en nombre la haute fonction publique.

Le PRM commence alors à formuler ses propres exigences. Il réclame deux postes ministériels et cinq secrétariats d'État. Le Premier ministre reste sourd à cette requête dans un premier temps, décidé à n'ouvrir le gouvernement aux partis anti-système que dans la stricte mesure du nécessaire. Il ne peut pour autant repousser un allié potentiel. Il est ainsi placé sur la corde raide : il entretient le dialogue avec Tudor aussi longtemps que possible, en prenant garde de ne jamais 
formuler des propositions trop précises. Cette stratégie tourne court : en décembre 1994, le PRM annonce son intention de voter toute motion de censure déposée contre le gouvernement.

En catastrophe, le PDSR se résout à conclure un « protocole quadripartite » avec le PUNR, le PRM et le PSM 14. Il attache un soin particulier à la rédaction du texte d'accord. Les signataires promettent d'œuvrer à la « stabilité politique et sociale » du pays. Ils s'engagent à prendre en compte les « demandes légitimes » des minorités (le qualificatif est assez vague pour prêter aux interprétations les plus diverses). Ils se déclarent résolus à lutter « contre toute manifestation chauvine, raciste, anti-roumaine, antisémite, totalitaire, extrémiste, fasciste ou de caractère nazi » (le terme « anti-roumaine » fait figure d'intrus ; il est inséré à la demande du PRM). Aucune clause ne précise que les partis alliés doivent tous disposer de postes ministériels. Seuls les termes de « collaboration » et de « consultation » sont employés. Le PDSR n'offre au PRM que trois secrétariats d'État et une préfecture.

Le Premier ministre et le président de la République tentent de prévenir les attaques extérieures par un déploiement d'explications. Ils précisent qu'ils s'appuient sur les membres les plus respectables des partis anti-système, mais non nécessairement sur leurs dirigeants ; en d'autres termes, ils entendent traiter avec les factions légalistes et refuser tout contact avec les factions séditieuses. Ils affirment par ailleurs que les nouveaux alliés du PDSR seront tenus en bride et assurent ne s'appuyer sur eux que pour se donner les moyens d'appliquer encore leur politique de réformes tempérées.

Ces arguments ne portent pas véritablement. L'opposition libérale dénonce avec virulence la formation d'un «quadrilatère rouge » (patrulater rosu). À l'intérieur du PDSR, la faction modérée dirigée par Melescanu commence de s'agiter. Sur la scène internationale, le gouvernement roumain est la cible de critiques nombreuses. Le Sénat américain condamne ouvertement le protocole quadripartite (il y est d'autant plus enclin que le PUNR multiplie les attaques à teneur antisémite contre Alfred Moses, nouvel ambassadeur américain à Bucarest, par ailleurs président de l'American Jewish Comittee). L'Internationale Socialiste retire au PDSR son statut d'observateur et intègre comme représentant de la Roumanie le Parti démocrate de Petre Roman. Le PDSR est également écarté du groupe socialiste à l'Assemblée parlementaire du Conseil de l'Europe.

S'il perturbe le PDSR, le protocole quadripartite sème également la discorde au sein des partis anti-système - et c'est là une contrepartie logique du pouvoir

14. Les relations entre le pouvoir et les partis anti-système sont déjà anciennes mais sont restées distendues jusque là : si un protocole de partenariat a été signé avec le PUNR en janvier 1994, il n'a fait que formaliser le principe du soutien sans condition et n'a guère suscité de polémique. 
parasitaire. Les représentants des factions légalistes sont seuls intégrés au gouvernement. Les factions séditieuses, pourtant majoritaires au sein du PUNR et du PRM, demeurent à l'écart. Elles sont jugées indésirables par le PDSR. Ellesmêmes restent campées sur leurs positions et continuent de juger contre-productive la collaboration avec les partis à vocation majoritaire car elles entendent toujours renverser l'ordre établi par une attaque frontale. Elles se livrent à tous les débordements verbaux, formulent des revendications maximalistes et n'hésitent pas à critiquer ouvertement les décisions du gouvernement.

Funar réclame notamment que l'UDMR soit mise hors la loi : il l'accuse de violer l'article ler de la Constitution, selon lequel « la Roumanie est un État national souverain, indépendant, unitaire et indivisible ». Soucieux de ménager son allié, le gouvernement n'oppose pas un refus catégorique à cette demande. Les autorités hongroises décident alors de rompre les négociations engagées avec Bucarest pour la conclusion d'un traité de bon voisinage. Ce traité étant posé comme condition à l'entrée de la Roumanie dans l'OTAN, le gouvernement est finalement amené à condamner les déclarations de Funar. La faction séditieuse du PUNR s'essaye à la surenchère. Elle demande pêle-mêle l'instauration de l'état d'urgence, la condamnation immédiate de tous les Magyars qui possèdent un drapeau hongrois ou qui entonnent l'hymne hongrois, l'obligation pour tous les fonctionnaires magyars de passer un test de connaissance de la langue roumaine, l'éviction des Magyars de l'Armée, du Ministère de l'intérieur, de l'appareil judiciaire et des services secrets... Funar multiplie par ailleurs les lettres ouvertes au gouvernement. Le PDSR demande à l'appareil dirigeant du PUNR de se démarquer de son dirigeant. Le bureau permanent du PUNR se réunit en urgence. Faction légaliste et faction séditieuse s'y affrontent violemment. Le parti menaçant d'éclater, Funar doit en rabattre. Un message conciliant est finalement publié : le PUNR déclare « regretter » ses récents écarts de conduite et vouloir contribuer au succès du gouvernement. Cet apaisement n'est que provisoire. Funar continue de se répandre en déclarations au vitriol et menace de faire basculer la majorité. Il contraint ainsi le Premier ministre à de pénibles contorsions.

Du reste, le PRM se dresse à son tour sur ses ergots. Il se déclare insatisfait de la faible place qui lui est faite dans le gouvernement et réclame 50 postes supplémentaires dans les ministères et le corps préfectoral. Ses dirigeants attaquent le Premier ministre dans les termes les plus vifs. En octobre 1995, un « éditorial » retentissant est publié dans România Mare. Il est signé Alcibiade, pseudonyme sous lequel chacun reconnaît Corneliu Vadim Tudor. Le président de la République est directement pris pour cible. Il est accusé d'être un ancien membre du KGB et d'avoir « assassiné Ceausescu le jour saint de Noël ». Il est soupçonné de cacher des origines tziganes et d'être incapable par conséquent de comprendre le « génie roumain ». Il est décrit comme «vendu aux Juifs » (il a visité le mémorial de l'Holocauste lors d'un déplacement à Washington en 1993). La conclusion du réquisitoire est proférée sur le ton le plus sentencieux : « les Juifs t'on amené au pouvoir, tu restes du côté des Juifs ; tu n'as pas la moindre idée de la passion de Jésus-Christ et de la tragédie du peuple Roumain ». 
De nouveau, le PDSR demande au parti concerné s'il avalise les propos de son chef. La faction séditieuse reste très majoritaire au sein du PRM (les opposants légalistes, Moldovan et Stoiescu, ont du reste quitté le parti quelques mois plus tôt). Aussi le bureau permanent se solidarise-t-il massivement avec Tudor. Un seul de ses membre, Mircea Hamza, donne sa démission. Le PRM est alors exclu du gouvernement. Il choisit logiquement de ne plus le soutenir. Les dirigeants en place ne disposent plus d'une majorité parlementaire.

Ces troubles intérieurs se combinent avec des difficultés extérieures croissantes. En juin 1995, au sommet européen de Cannes, la Roumanie a posé sa candidature à l'adhésion à l'Union européenne. En décembre, lors du sommet de Luxembourg, cette candidature est repoussée. La frustration est d'autant plus vive que les chefs d'État européens distinguent officiellement deux blocs en Europe centrale et orientale. Bien que la Commission précise qu'aucun candidat ne remplit les critères d'adhésion, les pays du groupe de Visegrad se voient promettre de figurer dans une " première vague » d'élargissement, la Roumanie étant renvoyée à des perspectives plus lointaines, en compagnie de la Bulgarie, de la Slovaquie, de la Lituanie et de l'Estonie. Le Premier ministre parle d'une « discrimination » infondée, explicable seulement par un défaut de perception. Il estime que le protocole quadripartite, mal interprété, lui a injustement porté préjudice.

Pour prévenir de nouvelles déconvenues, et dans la perspective d'une prochaine entrée dans l'OTAN, le gouvernement s'attache alors à dissiper les soupçons que les chancelleries occidentales font peser sur lui 15. Il s'efforce d'arriver au plus vite à la signature d'un Traité d'entente, de coopération et de bon voisinage avec la Hongrie. Le 16 septembre 1996 à Timisoara, la Hongrie et la Roumanie paraphent un texte commun. L'arrivée des socialistes hongrois au pouvoir a facilité les négociations : à la différence de ses prédécesseurs, Gyulya Horn ne s'oppose pas à la reconnaissance d'une intangibilité des frontières. En contrepartie, Bucarest accepte que le Traité fasse mention de la recommandation 1201 du Conseil de l'Europe, relative aux droits linguistiques et culturels des minorités nationales.

15. En raison d'une russophobie prononcée, les partis anti-système sont assez embarrassés par la question de l'entrée dans l'OTAN. Refuser d'adhérer à l'Alliance atlantique, c'est se placer sous la menace de «l'impérialisme russe ». Pour les dirigeants du PUNR, il s'agit d'éviter que la Roumanie se trouve « dans une situation encore plus dangereuse qu'entre 1939 et 1944 »; " entre deux maux, la Russie et l'OTAN, il faut choisir le moindre, à savoir l'OTAN » (PUNR, 1997b). Dans le même temps, certains dénoncent un projet de « yougoslavisation » de la Roumanie et évoquent une entente tacite entre l'Allemagne, la Russie et les pays du groupe de Visegrad pour laisser Bucarest sur la touche... Lorsqu'en janvier 1994 la Roumanie adhère au Partenariat pour la Paix (moins de 15 jours après que l'OTAN en a énoncé le principe), tous les partis applaudissent : une coopération avec l'Alliance est offerte sans garanties de sécurité immédiate mais avec la perspective d'une intégration future, aucune discrimination n'étant du reste opérée entre les anciennes démocraties populaires. En revanche, lorsque l'OTAN délimite deux cercles d'élargissement et relègue la Roumanie dans le second, l'hypothèse du complot international ressurgit et les partis anti-système manifestent une plus grande inimitié envers les puissances atlantiques. 
Pour conforter ce succès diplomatique et pour retrouver une pleine crédibilité internationale, le PDSR doit encore recentrer sa politique intérieure. Il s'efforce de compenser par des réformes ostentatoires les effets désastreux de sa compromission avec les partis anti-système. Il ouvre ses portes à de nouvelles personnalités, ouvertement hostiles aux partis anti-système et attachées à entretenir un dialogue courtois avec les créditeurs internationaux. Parmi ces recrues, on compte Iosif Boda, ancien ambassadeur de Roumanie en Suisse, Marian Enache, ancien ambassadeur de Roumanie en Moldavie, Mircea Cosea, éminent économiste, et Viorel Salagean, ancien président de la commission des Finances au Sénat, rédacteur en chef de Adevarul economic (La vérité économique). Tous rejoignent au sein du parti la faction modérée constituée autour de Teodor Melescanu.

Le PUNR prend ombrage de ce redressement de trajectoire. Le Traité de bon voisinage à pour conséquence directe l'ouverture d'un consulat hongrois à Cluj. Toujours maire de cette ville, Funar accuse le président Iliescu d'être vendu aux Hongrois. Il réclame que soit engagée contre lui une procédure de suspension pour « haute trahison » et exige qu'un référendum sur le Traité soit organisé le même jour que les élections nationales. Les représentants du PUNR qui siègent au gouvernement, tous membres de la faction légaliste, sont de nouveau gênés par les prises de position de leur dirigeant. Ils déclarent approuver le Traité à titre individuel.

Pour clarifier la situation, et dans le prolongement logique de son recentrage, le Premier ministre annonce un remaniement ministériel : trois ministres PUNR sont évincés (le quatrième, Aurel Novac, conserve son poste après avoir choisi de quitter le parti de Funar) ; les 5 préfets désignés en août 1994 sont également démis de leurs fonctions. Le PUNR connaît alors de vives tensions internes : la faction légaliste tient Funar pour responsable de la rupture avec le PDSR, contestant l'efficacité de sa stratégie d'opposition frontale. Dans la perspective des prochains scrutins, elle doit pourtant éviter de le critiquer au grand jour et accepter qu'il défende les couleurs de la formation.

Les élections se tiennent au mois de novembre. Elles consacrent la victoire du bloc d'orientation libérale. La CDR, le Parti démocrate et l'UDMR envoient ensemble 200 représentants à la Chambre des députés $(122+53+25)$ et 77 au Sénat $(53+23+11) 16$. Le PDSR obtient 91 sièges à la Chambre des députés et 41 au Sénat. Le bloc d'orientation anti-système, constitué désormais des seuls PRM et PUNR, dispose de 37 sièges à la chambre des députés $(19+18)$ et de 15 sièges au Sénat $(8+7)$. À l'élection présidentielle, Ion Iliescu est battu par le

16. Avant la tenue du scrutin, le Parti démocrate s'est allié au Parti Social Démocrate Roumain (Partidul Social Democrat Român - PSDR), petite formation sans assise électorale mais nantie d'un fort ancrage historique (elle a été fondée au milieu du XIXe siècle). C'est ainsi qu'a pris forme l'Union Sociale Démocrate (Uniunea Social-Democrata - USD). 
candidat de la CDR, Emil Constantinescu : il recueille 32,25\% des voix au premier tour et $45,57 \%$ au second.

Pour les partis anti-système, l'heure est aux bilans. Des rééquilibrages internes s'opèrent conformément aux logiques caractérisées plus haut. Pour des mobiles différents et selon des modalités diverses, les trois formations se détournent de l'option séditieuse. Fort de sa progression électorale et animé de nouvelles ambitions, le PRM tend à adopter une posture légaliste. Le PSM met son échec sur le compte des scissions et des divisions qui l'ont déchiré ; il reste fidèle à son orientation première et continue de rechercher une alliance avec un parti à vocation majoritaire. La direction du PUNR change de mains : Funar est renversé par la faction légaliste.

En dépit de ces évolutions, et au sein même de la nouvelle opposition, les partis anti-système continuent d'exercer un fort pouvoir parasitaire. De fait, le PDSR est lui aussi en proie à quelques troubles. Après son échec électoral, Ion Iliescu est immédiatement porté à la présidence du parti. Adrian Nastase, ancien président de la Chambre des députés et réputé proche des partis anti-système, est désigné pour le seconder. La faction Melescanu s'insurge contre cette reprise en main. Elle met en cause l'alliance passée avec les partis anti-système et affirme que le redressement final de trajectoire n'a pas suffi à effacer la compromission des mois passés. Dans une logique de refondation, elle entend que les critiques formulées contre les excès du libéralisme soient conciliées avec une orientation plus « moderniste » et que le PDSR s'aligne sur les partis de centre gauche occidentaux.

Forte de ces principes, elle tente de prendre la direction du parti. La fédération de Sibiu lui sert tout d'abord d'appui. Au printemps 1997, plusieurs de ses délégués demandent que Ion Iliescu soit mis sur la touche. Au sein de l'appareil dirigeant, Iosif Boda réclame ensuite le départ d'Adrian Nastase et la désignation de Teodor Melescanu au poste de Vice-président. Ion Iliescu exige aussitôt sa démission mais la direction du parti se contente de lui adresser un blâme. Melescanu prend la défense de son collègue ; il demande que « chacun soit autorisé à exprimer son opinion ». En juin 1997, à l'approche de la conférence nationale du PDSR, Boda renouvelle son appel à un changement de ligne politique. Il est exclu du parti. Pour avoir multiplié les éditoriaux hostiles à Ion Iliescu, Viorel Salagean connaît le même sort. En signe de solidarité, Marian Enache présente lui-même sa démission.

La faction Melescanu se trouve numériquement amoindrie. Le 18 juin, ceux de ses membres qui demeurent au sein de l'appareil dirigeant forment un « groupe d'opinion pour la transformation du PDSR en un parti moderne et crédible, porteur d'un modèle de réforme alternatif, adapté aux besoins de la population ». Ils publient une Déclaration dans laquelle ils réclament une « restructuration radicale » du parti. Ils exigent la démission des dirigeants qui, en se montrant trop conciliants avec les formations anti-système, ont entaché l'image du PDSR dans l'opinion publique et sur la scène internationale (ApR, 1997a). 
Lors de la conférence nationale, le « groupe d'opinion » n'est pas autorisé à présenter une motion. Pour contrecarrer la fronde, la direction du PDSR se dote d'un nouveau programme qui laisse une place à l'autocritique. Le récent revers électoral est imputé à l'image négative qu'ont donné du parti certains cadres corrompus. Un doigt accusateur est néanmoins pointé vers « ceux qui distordent les réalités roumaines et jettent un discrédit injuste sur le peuple roumain » (la nouvelle majorité est implicitement visée). Pour « faire pièce à leur propagande », le principe doit être réaffirmé selon lequel « la Roumanie est un État national, indivisible et unitaire (...). Parce que son histoire le lui impose, le peuple roumain doit porter haut cette vérité et la transmettre aux générations futures. C'est son plus grand devoir ». " Le PDSR entend mettre au point les instruments qui permettront de capitaliser les intérêts nationaux de la Roumanie et de bloquer toute entreprise portant atteinte à l'identité nationale ».

Ces principes posés, Ion Iliescu et ses fidèles s'efforcent de raffermir leur doctrine. Ils définissent le PDSR comme un « parti social-démocrate, de centre gauche, moderne et populaire ». Ils réaffirment que les solutions libérales sont « inadaptées aux réalités roumaines » et taxent leurs promoteurs d' « aventurisme ». Ils expliquent que " la privatisation ne doit pas conduire au démembrement de l'économie roumaine ni à l'annihilation des industries nationales ». Ils préconisent que « l'État intervienne activement dans l'économie, de manière à développer un système d'industries nationales, lui-même susceptible d'exploiter tous les avantages financiers de l'économie de marché ». Ils entendent « adapter les politiques économique et industrielle roumaines aux normes communautaires, mais sans oublier jamais l'intérêt national roumain »; la collaboration avec les créditeurs internationaux leur paraît acceptable dans la seule mesure où elle ne porte pas atteinte à la « dignité nationale » de la Roumanie (PDSR, 1997, pp. 3-4, 10, 15-23 ct 51-58).

Melescanu et Cosea estiment ces formules trop ambiguës et considèrent que l'aggiornamento du PDSR reste incomplet. N'étant pas parvenus à imposer les principes contenus dans leur Déclaration, ils démissionnent du parti et rejoignent leurs comparses déjà exclus. Ils entament des pourparlers avec le Parti démocrate mais jugent que leurs interlocuteurs sont trop fermement arrimés à un programme libéral (réciproquement, les amis de Petre Roman leur reprochent de ne pas l'être assez). Le 19 septembre 1997, ils forment donc un nouveau mouvement baptisé Alliance pour la Roumanie (Alianta pentru Romania - ApR) et dirigé par Melescanu.

L'ApR souhaite « concilier les principes sociaux-démocrates avec les principes de facture libérale ». Elle se déclare attachée à « la pluralité des formes de propriété ». Elle propose d'avancer vers l'Europe et les structures atlantiques sans occulter les réalités locales. Une adresse aux citoyens est ainsi formulée : « Nous devons participer aux grands processus qui se déploient aujourd'hui dans le monde mais en conservant notre spécificité roumaine ». L'ApR proclame sa farouche volonté de défendre "l'intérêt national » de la Roumanie. Pour que cette défense soit «crédible et efficace », elle juge nécessaire de se maintenir à 
distance aussi bien des orientations « cosmopolites » que du « nationalisme excessif et agressif »(ApR, 1997a, pp. 2 et $3 ; 1997 b$, pp. 1 et 2 ; 1997c, pp. 79 ; 1998, pp. 3-7) 17.

La scission de la faction Melescanu et la formation de l'ApR peuvent être interprétées comme des conséquences différées de l'alliance nouée en 1994 avec les partis anti-système. Alors même qu'il est passé dans l'opposition, le PDSR continue de subir le pouvoir parasitaire du PSM, du PUNR et du PRM. Se trouvant délesté de ses membres les plus modérés et par un singulier effet d'enchaînement, il en vient à graviter autour d'un axe nouveau : il est affecté d'un tropisme anti-système plus marqué que dans la période précédente. Dans le dessein de s'opposer efficacement à la politique du gouvernement et pour contrer les initiatives du bloc d'orientation libérale au Parlement, il se rapproche bientôt du PRM. Dans la mesure où ce dernier a lui-même opéré un léger mouvement de bascule entre option séditieuse et option légaliste, le croisement des trajectoires est somme toute logique.

Ion Iliescu et ses fidèles s'élèvent contre la politique qui vise à « imposer un schéma, un moule ou un modèle qui n'a rien à voir avec le contexte spécifique roumain ». Ils protestent contre la politique de « désindustrialisation » engagée par le gouvernement. Ils demandent que soit élaborée « pour la négociation avec les organismes internationaux et avec l'Union européenne une plate-forme qui reflète l'intérêt national ». Ils accusent la nouvelle majorité de se soumettre à « la tyrannie du temps court », sans jamais réfléchir à la stratégie de long terme qui permettrait à la Roumanie de «sortir du sous-développement ». Ils mettent en cause la politique qui consiste à « privatiser les profits et nationaliser les pertes au moyen de privatisations illégales, illogiques et corruptrices ». Ils jugent cette politique « antiéconomique, antisociale et contraire à l'intérêt national ». Ils récusent « l'idée selon laquelle l'expression de l'identité nationale serait un frein au processus d'intégration et de développement ». Ils font grief au gouvernement d' « accepter sans discernement les programmes imposés par le FMI et la Banque mondiale ». Ils demandent que soit mis un terme à la « politique de courbettes devant les étrangers (politica de ploconire in fata strainatatii), politique qui blesse la dignité nationale ». Ils s'engagent solennellement à «bloquer toute initiative qui porterait atteinte aux intérêts nationaux, à la dignité nationale et à l'identité nationale ». Ils promettent de « redonner à la Roumanie le prestige qu'elle mérite dans la communauté mondiale des nations » (PDSR, 1998c, pp. 1 et $3 ; 1998$ d, pp. 1,4 et $13 ; 1998 f$, pp. 3 et $9 ; 1999$ a, p. 2).

17. Si l'ApR se présente comme un mouvement régénéré, plusieurs courants existent encore en son sein. Les dirigeants qui ont longtemps appartenu au PDSR sont résolument hostiles à la majorité libérale. Ceux qui ont été intégrés seulement à la veille des élections de 1996 en sont plus proches ; ils tentent d'établir des ponts avec le Parti Démocrate. En juillet 1999 , Mircea Cosea quitte du reste l'ApR et rejoint une petite formation baptisée Union des Forces de Droite (Uniunea Fortelor de Dreapta - UFD). 
Sur le plan intérieur, le PDSR s'insurge également contre « l'escalade des manifestations antinationales ». Il opère un rapprochement entre la situation présente et celle de l'année 1940 : comme au début de la Seconde guerre mondiale, les dirigeants affichent selon lui « un patriotisme hypocrite ». C'est avec la « complicité » du président Constantinescu que la minorité magyare s'attache aujourd'hui à « démembrer l'État-Nation unitaire roumain ». Un « processus de désintégration de l'État national unitaire roumain » est à l'œuvre, alimenté « par l'activisme séparatiste de l'UDMR et par les pressions encore plus puissantes exercées depuis l'extérieur ». Il s'agit de «stopper ces entreprises périlleuses ». «Le PDSR n'accepte pas ni n'acceptera jamais de transiger de quelque façon que ce soit avec l'intégrité de l'État roumain ». « Le PDSR est favorable à la formation, au maintien et à la consolidation des identités culturelles minoritaires, mais refuse d'accepter que le problème du droit des minorités se transforme en instrument de dissolution de l'État (...) Le territoire roumain est un et indivisible. L'espace économique, social et militaire doit demeurer homogène et cohésif »(PDSR 1998b, pp. 1, 4-5 et $7 ; 1998$ e, pp. 1-2).

Cet emprunt du PDSR à la thématique des formations anti-système ne doit pas laisser conclure à une totale idylle entre les partisans d'Iliescu et les disciples de Tudor. À mesure que la nouvelle majorité montre son impuissance à sortir la Roumanie du marasme économique, et de nouvelles échéances électorales se profilant à l'horizon, les relations entre le PDSR et ses partenaires informels se font de plus en plus tendues.

En s'appuyant sur le versant légaliste de leur discours, les partis anti-système peuvent espérer emporter seuls la mise : ils s'efforcent de convaincre l'électorat que leur programme est cohérent et que, les politiques menées par le PDSR et par les partis d'orientation libérale ayant pareillement échoué, ils sont les seuls à offrir une alternative crédible. Pour se démarquer de ces alliés infidèles et pour contrer leurs manœuvres, le PDSR doit revenir à des positions plus mesurées. Il s'attache à souligner le caractère irresponsable de Tudor et de ses compagnons, dénonce la forme outrancière de leurs propositions, s'efforce de rehausser le jugement porté sur son propre passage au pouvoir et tente de faire valoir sa « culture de gouvernement ». Il doit pourtant veiller à ce que son discours ne se confonde point avec celui des partis de l'opposition. Aussi ne peut-il se résoudre à remodeler totalement son argumentaire et à renoncer définitivement à toute alliance avec les partis anti-système.

Deux événements rapprochés - une grève des mineurs et l'intervention alliée contre la Yougoslavie - permettent d'apprécier ce nouvel exercice d'équilibre.

Pour répondre aux exigences du FMI, Radu Berceanu, ministre de l'Industrie et du commerce, annonce en décembre 1998 que quatre mines de la vallée du Jiu (Câmpu Neag, Petrila Sud, Dâlja et Barbateni), génératrices d'un déficit global de 300 milliards de lei, cesseront d'être exploitées dans les plus brefs délais. Conduits par Miron Cozma et encouragés par Corneliu Vadim Tudor, 15000 mineurs entament une grève. Le 5 janvier, la Ligue du syndicat des mineurs 
de la vallée du Jiu envoie une délégation auprès du gouvernement. Elle réclame que chaque mineur licencié reçoive une indemnité de 10000 dollars et 2 hectares de terrain. Les négociations se poursuivent durant 15 jours mais les autorités refusent d'accéder aux exigences des grévistes. Les mineurs décident alors de se rendre tous à Bucarest. Le gouvernement ayant pris soin de bloquer les lignes de chemin de fer, ils se déplacent en car. En chemin, ils sont rejoints par les salariés des grandes entreprises publiques. Des grèves de soutien sont organisées dans tout le pays. Tudor annonce que la «bataille finale » est imminente ; il parle d'une « révolte de libération contre le joug de la mafia et des traîtres à la patrie » ou encore d'une «guerre contre le FMI ». Les mineurs parviennent à gagner Rîmnicu Vîlcea $(150 \mathrm{~km}$ de Bucarest). La capitale cède à la panique. Le président Constantinescu proclame l'état d'urgence. Les intellectuels signent des pétitions pour la « sauvegarde de l'État de droit ». Des contre-manifestations sont organisées à Timisoara et à Bucarest. Elles rassemblent 8000 à 10000 personnes et sont présentées comme une réaction de la « majorité silencieuse » aux « mouvement anti-réformes ».

Les mineurs exigent de négocier avec le Premier ministre en personne. Devant la menace, Radu Vasile doit satisfaire cette revendication. Les deux parties se réunissent dans le monastère de Cozia. Le gouvernement cède quasiment tout le terrain qu'il a défendu durant trois semaines. Particulièrement satisfaits d'avoir obtenu le maintien en activité de deux mines menacées (Dâlja et Barbateni), les grévistes prennent le chemin du retour. Pour " couper court aux supputations ", Cozma démissionne du PRM. Il est arrêté quelques semaines plus tard et condamné à 18 ans de prison pour les violences dont il s'est rendu coupable lors de précédentes « descentes » sur la capitale.

Le PDSR appréhende avec embarras le mouvement des mineurs. Dans un premier temps, il soutient les grévistes et dénonce le traumatisme social provoqué par la politique libérale du gouvernement. Il se tient ensuite sur la réserve et se refuse à exploiter la situation. Il lui faut éviter de corroborer les accusations de la presse libérale, prompte à évoquer une collusion entre les grévistes, le PRM et les amis de Ion Iliescu (certains journaux n'hésitent pas à dénoncer un « complot national-communiste » préludant à un « acte de terrorisme insurrectionnel contre l'État et les citoyens »).

Les raids de l'OTAN sur la Yougoslavie placent également le PDSR dans une position inconfortable. Ils ont pour effet de revigorer temporairement les factions séditieuses au sein des parti anti-système. L'accession du Kosovo à l'indépendance paraissant envisageable, les dirigeants roumains craignent que la province de Voïvodine, où vivent 300000 Magyars, n'en vienne à formuler la même revendication ; par capillarité, les Magyars de Transylvanie pourraient être amenés à les imiter. En Hongrie, le Parti des petits propriétaires et le Parti de la vérité en appellent à la formation d'un État de Voïvodine indépendant. Le ministre hongrois des Affaires étrangères, Zsolt Nemeth, déclare que « si l'UDMR choisit de revendiquer une autonomie [pour les Magyars de Transylvanie], le gouvernement hongrois étudiera les modalités d'un soutien ». L'ambassadeur de Hongrie 
aux États-Unis évoque la possibilité de voir se reformer bientôt une « Grande Hongrie ». Le PRM trouve là une occasion de remettre au goût du jour son discours anti-magyar. Tudor explique qu'un conflit comparable à celui qui déchire la Yougoslavie menace la Transylvanie ; il affirme que les Magyars sont organisés sur le même mode que l'Armée de Libération du Kosovo et qu'ils « cachent une grande quantité d'armes ».

Le PDSR adopte alors une attitude des plus empruntée. Il est hostile à l'intervention mais redoute d'être placé sur le même plan que les partis anti-système. Il entend se montrer plus responsable que le PRM et se refuse à proférer des diatribes anti-occidentales. Il finit par livrer un propos on ne peut plus ambivalent : il déclare approuver l'intervention alliée mais réclame que soit respectée «l'intégrité territoriale de la Yougoslavie » et dénonce « l'escalade des projets révisionnistes en Europe ». Lors même qu'il est dans l'opposition et qu'il paraît libre de ses mouvements, le PDSR continue d'éprouver le pouvoir parasitaire des partis anti-système (PDSR, 1999d).

\section{CONCLUSION}

Il paraît désormais possible d'expliquer pourquoi et comment le PSM, le PUNR et le PRM parviennent à exercer une influence décisive sur la vie politique roumaine, en totale disproportion avec leur effectif militant et leur ancrage électoral. On peut admettre que par-devers leurs propositions confuses et leurs comportements visiblement erratiques, les trois partis sont mus par des ressorts identifiés. Ils occupent une place originale dans le système politique roumain. Ils n'y remplissent pas une « fonction tribunitienne », n'acceptent pas de demeurer en marge du « pouvoir actif » par simple calcul électoral et dans le souci de consolider leur assise politique, ne canalisent pas les élans protestataires de la population avec l'accord tacite et l'appui discret des partis à vocation majoritaire mais aspirent bel et bien à renverser l'ordre établi. Ils entendent exercer une « magistrature consulaire » pour bloquer la libéralisation économique du pays. Ils ne concourent pas à la stabilité du système politique roumain mais s'acharnent, au contraire, à le faire vaciller.

Le propos doit encore être précisé. C'est en raison même de leur propension au dédoublement doctrinal que les partis anti-système sont retenus d'exercer une «fonction tribunitienne ». Les factions séditieuses ne peuvent répondre aux crìtères définis par Georges Lavau puisqu'elles refusent de pactiser avec les partis à vocation majoritaire : elles entendent que les partis anti-système accèdent seuls au pouvoir, sans se compromettre par une quelconque alliance et en se contentant de fustiger les choix politiques du gouvernement ; elles déstabilisent le PDSR plutôt qu'elles n'assurent sa mainmise sur le pouvoir. Les factions légalistes ne peuvent adopter une orientation « tribunitienne » pour une raison exactement symétrique : elles sont trop étroitement associées aux partis à vocation majoritaire et ne sont nullement tenues en marge du pouvoir. En formulant des propositions élaborées, elles parviennent à conclure un accord avec le PDSR et 
à intégrer le gouvernement. Elles entendent ainsi miner de l'intérieur l'édifice politique constitué. Les deux caractères pourraient s'annuler si les partis antisystème étaient soucieux de cohésion et s'ils s'attachaient à défendre continûment la même ligne politique. Une synthèse devrait être opérée entre les approches séditieuse et légaliste. L'exercice d'une "fonction tribunitienne » pourrait être regardé comme une solution acceptable : il permettrait de soutenir l'ordre établi tout en le critiquant. Aucun compromis n'étant jamais recherché dans les faits, les factions séditieuse et légaliste affirment sans retenue leur singularité et s'y enferment réciproquement, chacune retenant l'autre d'adopter une logique «tribunitienne».

Une telle disposition n'est pas sans présenter quelques avantages : le PSM, le PUNR et le PRM peuvent attaquer frontalement l'ordre politique institué et s'efforcer de le pénétrer dans le même temps. Il parviennent ainsi à faire pression sur le PDSR. Les responsables de ce parti se trouvent placés dans une situation extrêmement inconfortable. Ils sont tentés de passer alliance avec les factions légalistes mais ne peuvent y parvenir sans se compromettre dans le même temps avec les factions séditieuses. Ils éprouvent le pouvoir parasitaire des partis antisystème aussi bien lorsqu'ils sont aux affaires (contorsions nécessaires pour former une majorité sans nouer une alliance compromettante) que lorsqu'ils se trouvent dans l'opposition (tiraillements entre souci d'efficacité et quête de respectabilité, tensions internes et scission).

Le dédoublement doctrinal peut cependant exposer les partis anti-système à quelques troubles. Des plus efficace lorsqu'il s'agit de faire pression sur un parti à vocation majoritaire, il devient un facteur d'affaiblissement lorsque la logique est poussée à son terme. Les factions légalistes passent-elles une alliance en bonne et due forme avec le PDSR et intègrent-elles le gouvernement que les factions séditieuses sont portées à radicaliser un peu plus encore leur discours. Estimant toujours que le système politique institué doit être combattu de l'extérieur, elles s'en prennent violemment aux dirigeants en place et se livrent à toutes les outrances. Par un effet de rétroaction, le PDSR est retenu de développer plus avant la collaboration avec les partis anti-système ; il fait en sorte qu'elle conserve un caractère simplement formel et se garde de donner des gages à ses partenaires. Dès cet instant, la faction légaliste est empêchée de mettre son programme à exécution : elle échoue à miner l'ordre établi de l'intérieur et ne peut tirer tous les fruits de sa participation au pouvoir. La logique du dédoublement doctrinal se retourne contre ceux-là mêmes qui en tiraient jusqu'alors le plus grand profit.

Les partis anti-système ne sont pas absolument maîtres de leur destin : le pouvoir parasitaire qu'ils parviennent à exercer ne saurait être décrit comme le résultat d'un choix souverain. Pour qu'il prenne toute son importance, une parfaite conjonction doit être observée entre variables internes et variables externes. Le dédoublement doctrinal doit atteindre une amplitude significative (les factions légaliste et séditieuse doivent être en mesure de s'exprimer) et le contexte politique doit offrir quelques points d'appui (un parti à vocation majoritaire doit se 
trouver dans une situation telle qu'une alliance ou un rapprochement idéologique avec les factions légalistes lui soit nécessaire). Les dirigeants des partis anti-système ne maîtrisent pas la seconde variable, de sorte que des problèmes de synchronisation peuvent être observés et que des dérapages incontrôlés sont toujours envisageables. Il n'en est que plus nécessaire d'analyser avec rigueur les structures internes et les positionnements externes du PSM, du PUNR et du PRM. 


\section{Références}

SOURCES PRIMAIRES (DOCUMENTS DIFFUSÉS PAR LES PARTIS POLITIQUES)

\section{Documents du PSM et du PS}

1992, Programul al PSM (Programme du PSM).

1996, De-atita grija pentru stinga, domnul Mohora sprijina dreapta-Adrian Paunescu, 12 novembrie 1996 ( $\grave{A}$ force de sollicitude pour la gauche, M. Mohora soutient la droite - Adrian Paunescu, 12 novembre 1996).

1997a, Conferinta de presa : Ilie Verdet, presedintele PSM, 25 februarie 1997 (Conférence de presse : Ilie Verdet, président du PSM, 25 février 1997).

1997b, Suntem hotarita sa continuam dialogul - Declaratia domnului llie Verdet, presedintele Partidului Socialist al muncii, 4 aprilie 1997 (Nous sommes décidés à poursuivre le dialogue - Déclaration de M. Ilie Verdet, président du PSM, 4 avril 1997).

1997c, Conferinta de presa din 29 iulie 1997 (Conférence de presse du 29 juillet 1997).

1997d, Conferinta de presa a Partidului Socialist din 23 septembrie 1997 (Conférence de presse du PS, 23 septembre 1997).

1999, Cu siguranta, viitorul apartine stîngii democrate - Ilie Verdet, 29 iulie 1999 (Assurément, l'avenir est à la gauche démocratique - Ilie Verdet, 29 juillet 1999).

\section{Documents du PUNR}

1992, Programul politic (Programme politique).

1997a, Declaratie de presa PUNR despre guvernul Ciorbea (Communiqué de presse du PUNR sur le gouvernement Ciorbea).

1997a, Statutul si programul politic al PUNR (Statut et programme politique du PUNR).

\section{Documents du PRM}

1993, Programul Partidului (Programme du parti).

1996a, Strategia Fulger (Stratégie éclair).

1996b, Doctrina Nationala (Doctrine nationale).

1997a, Doctrina Nationala (îmbunatatita) (Doctrine nationale : améliorations).

1997b, Evenimente politice (Événements politiques).

1997c, Programul de guvernare : Unire în Belsug (Programme de gouvernement : l'Union dans l'Abondance).

1999a, Programul Anticriza (Programme anti-crise).

1999b, Economistii PRM lanseaza programul de guvernare - Biroul de Presa al Partidului România Mare, 23 februarie 1999 (Les économistes du PRM proposent un programme de gouvernement - Bureau de presse du PRM, 29 février 1999).

1999c, Organizatia de Tineret Romania Mare : Organizare si obiective generale ; programme si activitatii (Organisation de la Jeunesse de la Grande Roumanie : Organisation et objectifs généraux ; programme et activités). 


\section{Documents du PDSR}

1997, Programul politic al Partidului Democratiei Sociale din România : o noua realitate, un nou program (adoptat la Conferinta nationala din 20-27 iunie 1997) (Programme politique du PDSR : une nouvelle réalité, un nouveau programme adopté lors de la Conférence nationale du 20-27 juin 1997).

1998a, Conceptia de reforma in sprijinul doctrinei social-democrate, prezentata de dna. Hildegard Puwak - consiliul national, Bucuresti, 3 septembrie 1998 (Définition de la réforme permettant d'appuyer la doctrine sociale-démocrate, présentée par $\mathbf{M m e ~} \mathrm{H}$. Puwak, Conseil national, Bucarest, 3 septembre 1998).

1998b, Pozitia Partidului Democratiei Sociale din România privind grava criza politica, economica si sociala din România - discursul domnului Ion Iliescu, Presedinte al PDSR - Consiliul National, Bucuresti, 2 octombrie 1998 (La position du PDSR sur la grave crise politique, économique et sociale que traverse la Roumanie - discours de $M$. Ion Iliescu, président du PDSR, Conseil national, Bucarest, le 2 octobre 1998).

1998c, Declaratia biroului executiv central al Partidului Democratiei Sociale din România - 15 ianuarie 1998 (Déclaration du bureau exécutif central du PDSR, 15 janvier 1998).

1998d, Alternativa de Guvernare a Partidului Democratiei Sociale din Romania - Un program realist pentru salvarea României - Asa nu se poate! Sa prevenim dezastrul! - Consilul national, Bucuresti, 2 octombrie 1998 (Le projet de gouvernement alternatif du PDSR - un programme réaliste pour le salut de la Roumanie - Cela ne peut plus durer ! Prévenons le désastre - Conseil national, Bucarest, le 2 octobre 1998).

1998e, Declaratia Partidului Democratiei Sociale din România : cu privire la escaladarea manifestarilor antinationale, prezentata de dl. Liviu Maior - Consiliul national, Bucuresti, 2 octombrie 1998 (Déclaration du PDSR sur l'escalade des manifestations anti-nationales, présentée par M. Liviu Maior - Conseil national, Bucarest, le 2 octobre 1998).

1998f, Politica externa a României - Consiliul national, Bucuresti, 3 septembrie 1998 (La politique étrangère de la Roumanie - Conseil national, Bucarest, le 3 septembre 1998).

1998g, Oferta PDSR pentru salvarea României - Sa schimbam schimbarea! (Propositions du PDSR pour le salut de la Roumanie - Changeons le changement !).

1999a, Strategia PDSR privînd actuala criza politica, economica si sociala din România (Stratégie du PDSR face à la crise politique, économique et sociale traversée actuellement par la Roumanie).

1999b, Programul anticriza al PDSR (Programme anti-crise du PDSR).

1999c, Resolutia Consiliului National al PDSR privînd consolidarea unitatii, suveranitatii si independentiei României, apararea interesului national (Résolution du Conseil national du PDSR sur la consolidation de l'unité, de la souveraineté et de l'indépendance de la Roumanie et sur la défense de l'intérêt national).

1999d, Declaratia de presa in legatura cu ultimele evolutii privind criza din Kosovo, 30 martie 1999 (Déclaration de presse sur les dernières évolutions de la crise du Kosovo, 30 mars 1999).

\section{Documents de l'ApR}

1997a, Declaratie grupului de opinie pentru transformarea PDSR intr-un partid modern si credibil, cu o alternativa credibila de reforma, in interesul populatiei (Déclaration du groupe d'opinion pour la transformation du PDSR en un parti réformateur moderne et crédible, dans l'intérêt de la population). 


\section{ANTOINE ROGER}

1997b, Scurt istoric : inceracarea de reformare a PDSR, nastera Alientiei pentru Romania, prioritati ale Aliantiei pentru Romania (Bref historique : tentatives de réforme du PDSR, naissance de l'ApR, priorités de l'ApR pour la Roumanie).

1997c, Exista solutii : programul ApR (Bucuresti, 6 decembrie 1997) (Il existe des solutions : programme de l'ApR - Bucarest, 6 décembre 1997).

1998, Program de privatizare si restructurare economica (Programme de privatisation et de restructuration économique).

Alianta (Buletinul ApR), 5 janvier 1998.

Alianta, (Buletinul ApR), 9 janvier 1998. 


\section{SOURCES SECONDAIRES}

ANTOHI Sorin (1998), "Les Roumains pendant les années 90 : géographie symbolique et identité sociale", Transitions (Bruxelles), 29(1), pp. 111-134.

BoARI Mircea (1994), "Structural Limits of the Political Socialization in Romania: Case Study on the Political Discourse", Sfera Politici, n² 20, pp. 10-11.

Deletant Dennis (1991), "The Role of Vatra Romaneasca in Transylvania", Report on Eastern Europe, ler février, pp. 40-44.

Gallagher Tom (1992), "Vatra Româneasca and Resurgent Nationalism in Romania", Ethnic and Racial Studies, 15(4), pp. 570-598.

Gallagher Tom (1997), "Nationalism and Post-Communist Politics: The Party of Romanian National Unity, 1990-1996", in Lavinia Stan (dir.), Romania in Transition, Dartmouth : Aldershot, pp. 3-24.

Heath Anthony, Evans Geoffrey \& Marginean Ioan (1994), Clasa sociale si politica în Europa de est (Classes sociales et politique en Europe de l'Est), Revista de Cercetari Sociale, 1(2), pp. 59-67.

Ivan Gabriel (1993), Criza parlamentarismului (La crise du parlementarisme), Sfera Politicii, $\mathrm{n}^{\circ} 10$, pp. 6-7.

Lavau Georges (1981), À quoi sert le Parti Communiste Français ?, Paris : Fayard.

Minu Achim (1994), Fenomenul Caritas în contextul tranzitiei (Le phénomène Caritas dans le contexte de la transition), Revista de Cercetari Sociale, 1(3), pp. 107-120.

Neumann Victor (1993), Doctrina nationalista (la doctrine nationaliste), Sfera Politicii, $\mathrm{n}^{\circ} 2$, p. 12.

PePine Horatiu (1994), Partidul Unitatii National Române (Le Parti de l'unité nationale roumaine), Sfera Politicii, $\mathrm{n}^{\circ} 16$.

PiRvulescu Cristian R. (1993), Tipologia clasei politice în România postotalitara (Typologie de la classe politique dans la Roumanie post-totalitaire), Sfera Politicii, n 11 , pp. 9-10.

ROPER Steven D. (1995), "The Romanian Party System and the Catch-all Party Phenomenon", East European Quarterly, 28(4), pp. 519-532.

Sartori Giovanni (1976), Parties and Party Systems: A Framework for Analysis, Cambridge : Cambridge University Press.

Shafir Michael (1991), "The Greater Romania Party", Report on Eastern Europe, 15 novembre.

SHAFIR Michael (1993), "The Revival of Political Right in Post Communist Romania", in Joseph Held, (Ed.), Democracy and Right-Wing Politics in Eastern Europe in the 1990 s , New York : Columbia University Press, pp. 153-174.

SHAFIR Michael (1994), “The Inheritors: The Romanian Radical Right Since 1989”, East European Jewish Affairs, 24(1), pp. 71-89.

SHAFIR Michael (1999), "The Mind of Romania's Radical Right", in Sabrina Ramet (Ed.), The Radical Right in Central and Eastern Europe Since 1989, University Park : The Pennsylvania State University Press, pp. 213-232.

SmITH Gordon (1987), "Party and Protest: The Two Faces of Opposition in Western Europe", in Eva Kolinski (Ed.), Opposition in Western Europe, London : Croom Helm, pp. 49-72.

Stefan-Scalat Laurentiu (1999), Partidul România Mare (Le Parti de la Grande Roumanie), Sfera Politicii, ${ }^{\circ}$ 67, pp. 4-5.

StOENESCU Alex Mihai (1998), "Problema evreiasca" astazi (Le "problème juif" aujourd'hui), Sfera Politicii, n 60, pp. 4-5. 
VEIGA Francisco (1997), "On the Social Origins of Ultranationalism and Radicalism in Romania, 1989-1993", in Lavinia Stan (dir.), Romania in Transition, Aldershot : Dartmouth, pp. 49-66.

VoICu George (1993a), Discursul nationalist (Le discours nationaliste), Sfera Politicii, $n^{\circ} 5$, pp. 4-5 (1 ère partie) ; $n^{\circ} 7$, pp. 20-21 (2e partie).

VoICu George (1993b), Sistemul de partide în România postcomunista (Le système de partis de la Roumanie post-communiste), Sfera Politicii, ${ }^{\circ} 3,1993$, pp. 3-4. 